\title{
Synchronization, chaos, and breakdown of collective domain oscillations in reaction-diffusion systems
}

\author{
C. B. Muratov \\ Department of Physics, Boston University, Boston, Massachusetts, 02215
}

(November 10, 2018)

\begin{abstract}
The universal equations describing collective oscillations of the multidomain patterns of small period in an arbitrary $d$-dimensional reaction-diffusion system of the activator-inhibitor type are asymptotically derived. It is shown that not far from the instability leading to the formation of the pulsating multidomain pattern the oscillations of different domains synchronize. In one dimension standing and traveling waves of the oscillation phase are realized. In addition to these, in two dimensions target and spiral waves of the oscillation phase, as well as spatio-temporal chaos of domain oscillations, are feasible. Further inside the unstable region the collective oscillations break down, so the pulsating multidomain pattern transforms into an irregular pulsating pattern, the uniform self-oscillations, or turbulence. The parameter regions where these effects occur are analyzed. The effects of the pattern's disorder are also studied. The conclusions of the analysis are supported by the numerical simulations of a concrete model. The obtained results explain the dynamics of Turing patterns observed in the experiments on chlorite-iodide-malonic acid reaction.
\end{abstract}

PACS number(s): 05.70.Ln, 82.20.Mj, 47.54.+r

\section{INTRODUCTION}

Complex dynamic patterns, such as traveling waves, breathers, or spatio-temporal chaos, are encountered in the variety of nonequilibrium physical, chemical, and biological systems [1 11]. These systems include electronhole and gas plasma, semiconductor and superconductor structures, systems with uniformly generated combustion material, autocatalytic chemical reactions, models of population dynamics 22 11. Recently, an intriguing phenomenon was observed in the chemical experiments with Turing patterns [12], combustion system with cellular flames [13], and the catalytic reaction on the surface [14]. In these experiments the patterns that were observed consisted of many disk-shaped domains whose radii oscillated in time. The oscillations of different domains either synchronized or exhibited complex spatiotemporal behavior.

Many nonequilibrium systems in which patterns can form, including the systems mentioned above, are described by the reaction-diffusion systems of the activatorinhibitor type, the simplest of which is a pair of reactiondiffusion equations [2 11]

$$
\begin{gathered}
\tau_{\theta} \frac{\partial \theta}{\partial t}=l^{2} \Delta \theta-q(\theta, \eta, A), \\
\tau_{\eta} \frac{\partial \eta}{\partial t}=L^{2} \Delta \eta-Q(\theta, \eta, A),
\end{gathered}
$$

where $\theta$ is the activator, $\eta$ is the inhibitor, $l$ and $L$ are the characteristic length scales and $\tau_{\theta}$ and $\tau_{\eta}$ are the characteristic time scales of the activator and the inhibitor, respectively; $q$ and $Q$ are certain non-linear functions, and $A$ is the system's excitation level. For example, in the system with the uniformly generated combustion material the activator is the temperature of the gas mixture, the inhibitor is the density of the fuel, $A$ is proportional to the rate of the fuel supply, and the non-linear functions $q$ and $Q$ contain the dissipation, supply, and reaction terms [8,9. The length scales $l$ and $L$ are related to the diffusion coefficients of the activator and the inhibitor $D_{\theta}$ and $D_{\eta}$, respectively: $l=\sqrt{D_{\theta} \tau_{\theta}}$ and $L=\sqrt{D_{\eta} \tau_{\eta}}$.

Kerner and Osipov showed that the properties of the patterns forming in the systems described by Eqs. (1) and (2) are determined mainly by the parameters $\epsilon=l / L$ and $\alpha=\tau_{\theta} / \tau_{\eta}$ and the form of the nullcline of Eq. (1). For many systems this nullcline is $\mathrm{N}$-shaped (Fig. 11) [8-10]. In such $\mathrm{N}$ systems static domain patterns form when $\epsilon \ll 1$ and $\alpha \gg \epsilon$ (KN systems), traveling waves (autowaves) at $\alpha \ll 1$ and $\alpha \lesssim \epsilon^{2}$ ( $\Omega \mathrm{N}$ systems), and all sorts of dynamic patterns at $\epsilon \ll 1$ and $\epsilon^{2} \lesssim \alpha \lesssim \epsilon$ (K $\Omega N$ systems) [8 10,15,16]. As a result of the instability of the homogeneous state periodic and more complex patterns form in these system, whereas when the homogeneous state of the system is stable one can excite solitary patterns - autosolitons (AS) - by a sufficiently strong localized external stimulus. In KN systems these patterns are collections of static domains with sharp walls (interfaces) whose width is of order $l$ [8,9, 15, 16]. These domain patterns may undergo different kinds of instabilities leading to the formation of complex dynamic patterns when $\alpha$ becomes sufficiently small. The simplest example of such destabilization is the transformation of a static AS to a pulsating (breathing) AS. This effect was discovered by Koga and Kuramoto in an axiomatic reaction-diffusion model 17 and subsequently studied by many authors, both for one-dimensional and higherdimensional radially-symmetric AS [8 $10,15,18,19]$. Pulsating AS were directly observed in semiconductors [20], 
composite superconductors 21], autocatalytic reaction 22], and combustion experiments [23].

The situation becomes much more complicated when instead of a single AS the pattern consists of many interacting domains. Several attempts to approach this problem were made for one-dimensional N systems. Kerner and Osipov showed that the stationary periodic domain patterns (strata) may undergo instability and transform into a breathing pattern [8,10]. Ohta et al. were able to obtain the linearized equation of motion for the periodic patterns in the piecewise-linear reaction-diffusion model and showed that the introduction of a simple nonlinearity to these equations leads to the synchronization of the domain oscillations 24,25. Still, the question of the effect of the interaction of domain patterns undergoing breathing motion, especially in higher-dimensional systems, remains largely unresolved.

It is easy to show that in the case $\epsilon \ll 1$ the wave length of the fluctuation with respect to which the Turing instability of the homogeneous state of the system (11) and (2) is realized is $\lambda \sim(l L)^{1 / 2}$ [8, 10]. For this reason the periodic Turing structures that form in the system have the period $\mathcal{L}_{p} \sim \epsilon^{1 / 2} L \ll L$. Moreover, in the higher-dimensional systems with $\epsilon \ll 1$ any static pattern will consist of the domains whose characteristic size is of order $\epsilon^{1 / 3} L \ll L[15,16,26$. This means that in a pattern consisting of many domains one domain interacts with a large number of other domains at the same time. This fact should significantly reduce the complexity of the interactions between different domains.

In this paper we will study the periodic onedimensional and two-dimensional hexagonal patterns of small period $\left(\mathcal{L}_{p} \ll L\right)$ undergoing the oscillatory instability in an arbitrary $\mathrm{K} \Omega \mathrm{N}$ system. Using the interfacial dynamics approach, we will derive the universal nonlinear equations describing the pulsations of the periodic multidomain patterns in arbitrary dimensions. We will analyze the conditions for the synchronization and breakdown of the pulsations, the effects of the disorder, and study possible complex spatio-temporal behaviors. Finally, we will compare the effects studied by us with relevant experiments.

Our paper is organized as follows. In Sec. II we reduce Eqs. (11) and (2) to the problem of interfacial dynamics in the limit $\epsilon \rightarrow 0$ and show the way of treating this problem in the case $\mathcal{L}_{p} \ll 1$. In Sec. III we apply this method to the one-dimensional periodic strata. In Sec. IV we consider two-dimensional hexagonal patterns and also briefly discuss the case of the three-dimensional patterns. In Sec. V we analyze the domain oscillations in a disordered pattern with a random distribution of domain sizes. In Sec. VI we present the results of the numerical simulations for a concrete model, and, finally, in Sec. VII we discuss the relevancy of the obtained results to the experiments and draw conclusions.

\section{INTERFACIAL DYNAMICS PROBLEM}

Let us measure the length and time in the units of $L$ and $\tau_{\eta}$, respectively. Then Eqs. (1) and (2) become

$$
\begin{gathered}
\alpha \frac{\partial \theta}{\partial t}=\epsilon^{2} \Delta \theta-q(\theta, \eta, A), \\
\frac{\partial \eta}{\partial t}=\Delta \eta-Q(\theta, \eta, A) .
\end{gathered}
$$

The boundary conditions for these equations may be neutral or periodic. From the mathematical point of view the fact that $\theta$ is the activator and $\eta$ is the inhibitor means that for some values of $\theta$ and $\eta$ we have $q_{\theta}^{\prime}<0$, and that for all $\theta$ and $\eta \otimes 10,15$

$$
Q_{\eta}^{\prime}>0, \quad q_{\eta}^{\prime} Q_{\theta}^{\prime}<0
$$

and the derivatives $Q_{\eta}^{\prime}, Q_{\theta}^{\prime}$, and $q_{\eta}^{\prime}$ do not change sign.

The dynamics of the domain patterns forming in $\mathrm{KN}$ and $K \Omega N$ systems can be reduced to the interfacial dynamics problem in which the dynamics of the pattern interfaces is coupled to the bulk field 18,26. Far enough from the domain interfaces (at distances much greater than $\epsilon$ ) the bulk field must satisfy the equation of smooth distributions (outer solution)

$$
\frac{\partial \eta}{\partial t}=\Delta \eta-Q(\theta(\eta), \eta, A)
$$

in which $\theta$ and $\eta$ are related by the equation of local coupling

$$
q(\theta, \eta, A)=0
$$

that is, far from the domain interfaces $\theta$ and $\eta$ lie on the nullcline of Eq. (3). This relation is multivalued (see Fig. (1), so one has to choose the branch with $\theta<\theta_{0}$ in the "cold" regions (the domains of low values of $\theta$ ) and $\theta>\theta_{0}^{\prime}$ in the "hot" regions (the domains of high values of $\theta$ ). The dynamics of the interface is governed by the following equation

$$
\vec{n} \cdot \frac{\partial \vec{r}}{\partial t}=-\epsilon^{2} \alpha^{-1} K(\vec{r})+v(\eta(\vec{r})),
$$

where $\vec{r}$ is a point on the interface, $\vec{n}$ is the normal vector to the interface pointing into the cold region, $K(\vec{r})$ is the curvature at the point $\vec{r}$ of the interface, and $v(\eta(\vec{r}))$ is the velocity of the interface in the absence of the curvature as a function of the value of the bulk field $\eta(\vec{r})$ at the interface. The function $v(\eta)$ is a solution to the nonlinear eigenvalue problem and is in general some complicated nonlinear function of $\eta$ 18,26.

The interfacial dynamics problem represented by Eqs. (6) and (8) is a highly nonlinear problem. However, the situation becomes simpler if the pattern consists of the alternating hot and cold regions whose characteristic size 
is much smaller than 1 . Kerner and Osipov showed that in this case the value of $\eta$ is close to a constant in the entire pattern [8,10]. The reason is that because of its high diffusivity the inhibitor cannot react well on these variations of the activator whose characteristic length is much smaller than the characteristic length scale of the inhibitor. This allows us to linearize Eq. (6) around $\eta=\eta_{s}$, where $\eta_{s}$ is the constant value of the inhibitor. Introducing

$$
\tilde{\eta}=\eta-\eta_{s}
$$

we get

$$
\frac{\partial \tilde{\eta}}{\partial t}=\Delta \tilde{\eta}-c_{1} \tilde{\eta}-\left(c_{3}-c_{1}\right) \tilde{\eta} I(x)-Q\left(\theta_{s 1}, \eta_{s}\right)-a I(x)
$$

where

$$
\begin{gathered}
c_{1,3}=Q_{\eta}^{\prime}\left(\theta_{s 1,3}, \eta_{s}\right)-\frac{q_{\eta}^{\prime}\left(\theta_{s 1,3}, \eta_{s}\right) Q_{\theta}^{\prime}\left(\theta_{s 1,3}, \eta_{s}\right)}{q_{\theta}^{\prime}\left(\theta_{s 1,3}, \eta_{s}\right)} \\
a=Q\left(\theta_{s 3}, \eta_{s}\right)-Q\left(\theta_{s 1}, \eta_{s}\right)
\end{gathered}
$$

the values of $\theta_{s 1,3}$ satisfy

$$
q\left(\theta_{s 1,3}, \eta_{s}\right)=0
$$

$\theta_{s 1}$ and $\theta_{s 3}$ being the minimal and the maximal roots of Eq. (13), respectively; $I(x)$ is the indicator function which is equal to 1 if $x$ is in the hot region and zero in the cold region. Notice that, according to Eqs. (5), the constants $c_{1}$ and $c_{3}$ are positive.

Obviously, $\eta_{s}$ should be equal to the value of $\eta$ at which $v(\eta)=0$ in order for the pattern to be stationary. The value of $\eta_{s}$ must therefore satisfy [8,10,26]

$$
\int_{\theta_{s 1}}^{\theta_{s 3}} q\left(\theta, \eta_{s}\right) d \theta=0
$$

For small $\tilde{\eta}$ the function $v(\eta)$ may be linearized around $\eta_{s}$, so we obtain [26]

$$
v=\frac{\epsilon B \tilde{\eta}}{\alpha Z a}
$$

where

$$
B=-a \int_{\theta_{s 1}}^{\theta_{s 3}} q_{\eta}^{\prime}\left(\theta, \eta_{s}\right) d \theta
$$

and

$$
Z=\int_{\theta_{s 1}}^{\theta_{s 3}} \sqrt{-2 U_{\theta}} d \theta, \quad U_{\theta}=-\int_{\theta_{s 1}}^{\theta} q\left(\theta, \eta_{s}\right) d \theta .
$$

The constants $B$ and $Z$ are of order 1 . Notice that, according to Eq. (5), the value of $B$ is positive.
Although Eqs. (8) and (10) with (15) are simpler than the original interfacial dynamics equations, they are still difficult to deal with. It appears, however, that these equations can be further simplified for treating multidomain patterns by introducing some averaged variables. We will outline this procedure in this section and demonstrate its application to the periodic patterns in the subsequent sections.

Let us introduce the number of domains per unit volume $n$ and the radius of the domain $\rho$ (in one dimension $\rho$ is the half-width of a domain). If we now average Eq. (10) over the volume of size $s$ such that $\rho \lesssim s \ll 1$, we will obtain the "coarse-grained" equation for the average value of the inhibitor $\bar{\eta}=\langle\tilde{\eta}\rangle$

$$
\frac{\partial \bar{\eta}}{\partial t}=\Delta \bar{\eta}-c_{1} \bar{\eta}\left(1+c_{2} n \rho^{d} \Omega_{d}\right)-Q\left(\theta_{s 1}, \eta_{s}\right)-a n \rho^{d} \Omega_{d},
$$

where $\Omega_{d}$ is the volume of the $d$-dimensional unit sphere $\left(\Omega_{1}=2\right)$, and

$$
c_{2}=\left(c_{3}-c_{1}\right) / c_{1}
$$

measures the asymmetry between the hot and the cold domains. According to Eq. (5), the value of $c_{2}>-1$. Besides $\bar{\eta}$, there is a local contribution to $\tilde{\eta}$ due to the variation of $\tilde{\eta}$ on the length scales of $\rho \ll 1$. Let us introduce

$$
\hat{\eta}=\tilde{\eta}-\bar{\eta}
$$

Since $\hat{\eta}$ varies on the short length scales, the terms proportional to $\eta$ in the right-hand side of Eq. (10) are small compared to the Laplacian as well. Also, when the characteristic time scale of variation of $\hat{\eta}$ is much greater than $\rho^{2}$, as is the case in all interesting situations (see the following sections), the time derivative in Eq. (10) is small compared to the Laplacian. Subtracting Eq. (18) from Eq. (10) and neglecting all these terms, we obtain

$$
\Delta \hat{\eta}=a\{I(x)-\langle I(x)\rangle\}, \quad\langle\hat{\eta}\rangle=0 .
$$

From this equation one can obtain the value of $\hat{\eta}=\hat{\eta}_{s}$ in the wall of an individual domain, so from Eqs. (8) and (15) follows that the equation for the radius is

$$
\frac{\partial \rho}{\partial t}=-\frac{\epsilon^{2}(d-1)}{\alpha \rho}+\frac{\epsilon B\left(\bar{\eta}+\hat{\eta}_{s}\right)}{\alpha Z a} .
$$

The variables $\bar{\eta}, \hat{\eta}_{s}$, and $\rho$ may now be considered as space- and time-dependent on the length scales much greater than $s$. Thus, we have a closed set of equations for these coarse-grained variables, so the number of the relevant dynamical variables in the problem is considerably reduced. 


\section{ONE-DIMENSIONAL PERIODIC STRATA OF SMALL PERIOD}

Let us consider the one-dimensional periodic strata of the period $\mathcal{L}_{p} \ll 1$ (Fig. 2). In this case $n=\mathcal{L}_{p}^{-1}$, so Eq. (18) becomes

$$
\frac{\partial \bar{\eta}}{\partial t}=\frac{\partial^{2} \bar{\eta}}{\partial x^{2}}-c_{1} \bar{\eta}\left(1-2 c_{2} \mathcal{L}_{p}^{-1} \rho\right)-2 a \mathcal{L}_{p}^{-1}\left(\rho-\rho_{0}\right),
$$

where we introduced

$$
\rho_{0}=-\mathcal{L}_{p} Q\left(\theta_{s 1}, \eta_{s}\right) / 2 a .
$$

Equation (21) for a one-dimensional pattern with the pe$\operatorname{riod} \mathcal{L}_{p}$ becomes

$$
\frac{d^{2} \hat{\eta}}{d x^{2}}=a I(x)-2 a \mathcal{L}_{p}^{-1} \rho, \quad\langle\hat{\eta}\rangle=0
$$

with neutral boundary conditions at $x= \pm \mathcal{L}_{p} / 2$ (because of the translational invariance, we may choose the center of the domain to be at $x=0$ ). A straightforward calculation gives

$$
\hat{\eta}_{s}=a\left(-\frac{\rho \mathcal{L}_{p}}{6}+\rho^{2}-\frac{4 \rho^{3}}{3 \mathcal{L}_{p}}\right) .
$$

Rescaling the variables $\bar{\eta}$ and $\rho$

$$
\bar{\eta}^{\prime}=\bar{\eta} / a \mathcal{L}_{p}^{2}, \quad \bar{\rho}=\rho / \mathcal{L}_{p}
$$

and introducing the quantities

$$
\tau_{1}=\alpha Z / \epsilon B \mathcal{L}_{p}, \quad \omega_{0}=\sqrt{2 \epsilon B / \alpha Z \mathcal{L}_{p}}
$$

we write Eqs. (22) and (23) (dropping the primes) as

$$
\begin{gathered}
\frac{\partial \bar{\eta}}{\partial t}=\frac{\partial^{2} \bar{\eta}}{\partial x^{2}}-c_{1} \bar{\eta}\left(1+2 c_{2} \bar{\rho}\right)-\omega_{0}^{2} \tau_{1}\left(\bar{\rho}-\bar{\rho}_{0}\right), \\
\tau_{1} \frac{\partial \bar{\rho}}{\partial t}=\bar{\eta}-\frac{\bar{\rho}}{6}+\bar{\rho}^{2}-\frac{4 \bar{\rho}^{3}}{3} .
\end{gathered}
$$

The parameters of the original reaction-diffusion system [Eqs. (3) and (4)] enter these equations only through constants $\omega_{0}, \tau_{1}$, and $c_{2}$ (the value of $c_{1}$ can be absorbed in the definitions of $\tau_{1}$ and $\left.\omega_{0}\right)$, and the excitation level enters mainly through $\bar{\rho}_{0}$. Note that since, according to its definition, the value of $2 \rho$ cannot exceed $\mathcal{L}_{p}$, we have $0<\bar{\rho}<\frac{1}{2}$. Also note that Eqs. 29) and (30) with the spatial derivative equal to zero describe the oscillations of a single domain in the system of size $\mathcal{L}_{p} \ll 1$.

Equations of the type of Eqs. (29) and (30) have been extensively studied in the context of oscillatory chemical reactions [2 6, 27]. It was shown that these systems host a great richness of dynamic patterns, such as self-sustained uniform oscillations, traveling waves, target patterns and spirals (in higher dimensions), and spatio-temporal chaos (turbulence). This is also true for Eqs. (29) and (30). However, one should keep in mind that these equations describe the dynamics of the already existing multidomain pattern, in other words, the dynamical patterns described by Eqs. (29) and (30) will be seen "on top" of the stationary multidomain (Turing) patterns. This is an important distinction from the oscillatory systems, such as oscillatory chemical reactions, in which the dynamic patterns appear on top of the stationary homogeneous state.

We are interested in the collective oscillations of the domains in the pattern. According to Eqs. 29) and (30), the characteristic frequency of these oscillations is equal to $\omega_{0}$. In view of Eq. (28), the period of oscillations is the shortest time scale in the problem if

$$
\epsilon \mathcal{L}_{p}^{3} \ll \alpha \ll \epsilon \mathcal{L}_{p}^{-1} .
$$

Recall that in deriving Eq. (21) we neglected the time derivative of $\hat{\eta}$. This is justified if $\omega_{0} \ll \mathcal{L}_{p}^{-2}$, what is equivalent to $\alpha \gg \epsilon \mathcal{L}_{p}^{3}$, so the first condition in Eq. (31) is in fact a necessary condition for Eqs. (29) and (30) to be valid.

Equations (29) and (30) have a trivial solution $\bar{\rho}=$ const, $\bar{\eta}=$ const which corresponds to the stationary multidomain pattern (strata). For $\alpha$ satisfying the condition in Eq. (31) we have $\omega_{0} \gg 1$, so the value of $\bar{\rho}$ for the stationary pattern is close to $\bar{\rho}_{0}$. Linear stability analysis of Eqs. (29) and (30) shows that at $\tau_{1}>\tau_{1 c}$, where

$$
\tau_{1 c} \cong \frac{12 \bar{\rho}_{0}-24 \bar{\rho}_{0}^{2}-1}{6 c}
$$

and

$$
c=c_{1}\left(1+2 c_{2} \bar{\rho}_{0}\right)>0,
$$

this solution is stable. At $\tau_{1}=\tau_{1 c}$ it looses stability with respect to the uniform oscillations with the frequency $\omega \cong \omega_{0}$ and the wave vector $k=0$. The uniformly oscillating solution corresponds to the pattern in which all domains are oscillating in phase with the frequency $\omega_{0}$. This is a supercritical Hopf bifurcation (see below). In view of Eq. (28) and (32), the value of $\tau_{1 c} \sim 1$, so in terms of the original variables we have $\alpha_{c} \sim \epsilon \mathcal{L}_{p}$ and $\omega_{0} \sim \mathcal{L}_{p}^{-1}$. Also, according to Eq. (32), the value of $\tau_{1 c}>0$ only if

$$
\frac{1}{4}\left(1-\frac{1}{\sqrt{3}}\right)<\bar{\rho}_{0}<\frac{1}{4}\left(1+\frac{1}{\sqrt{3}}\right),
$$

i.e., $0.11<\bar{\rho}_{0}<0.39$. This is a completely universal result for all one-dimensional strata of small period in $\mathrm{K} \Omega \mathrm{N}$ systems. It is easy to see from Eq. (32) that if the condition of Eq. (34) is satisfied, for any acceptable value of $c_{2}$ there exists $\tau_{1 c}=\tau_{1 c}^{\max }$, such that the stationary pattern is stable for $\tau_{1}>\tau_{1 c}^{\max }$ for any value of $\bar{\rho}_{0}$. If the condition in Eq. (34) is not satisfied, the pattern will 
still become unstable when $\alpha \sim \epsilon^{2} \& 10,15$. This will happen at the values of $\alpha$ which are much smaller than in the former case. Indeed, according to Eq. (32), the oscillatory instability occurs at $\alpha \gg \epsilon^{2}$ since $\mathcal{L}_{p}$ is always much greater than $\epsilon$ [8, 10.

To study the destabilization in more detail we will use the fact that the period of the oscillations $\omega_{0}^{-1}$ is much smaller than the characteristic relaxation time $\tau_{1}$, so one can apply the method of Bogoliubov and Mitropolsky to Eqs. (29) and (30) 28. To do this, we will use Eq. (30) to eliminate $\bar{\eta}$ from Eq. (29) and write

$$
\bar{\rho}=\bar{\rho}_{0}+R \cos \left(\omega_{0} t+\Theta\right)
$$

where $R$ and $\Theta$ are slowly varying functions of $t$ and $x$. Substituting Eq. (35) into Eq. (29), multiplying it by $\sin \left(\omega_{0} t+\Theta\right)$ and $\cos \left(\omega_{0} t+\Theta\right)$ and integrating over the period, we will get, respectively,

$$
\frac{\partial R}{\partial t}=\frac{c R}{2 \tau_{1}}\left(\tau_{1 c}-\tau_{1}\right)-\frac{R^{3}}{2 \tau_{1}}+\frac{1}{2} \frac{\partial^{2} R}{\partial x^{2}}-\frac{R}{2}\left(\frac{\partial \Theta}{\partial x}\right)^{2},
$$

and

$$
\frac{\partial \Theta}{\partial t}=\frac{1}{2} \frac{\partial^{2} \Theta}{\partial x^{2}}+\frac{1}{R} \frac{\partial R}{\partial x} \frac{\partial \Theta}{\partial x} .
$$

In deriving Eqs. (36) and (37) we kept only the leading terms.

Equations. (36) and (37) are equivalent to the equations for the amplitude and phase for the well-known Newell-Whitehead equation [29]. An important class of solutions of this equation is $R=R_{0}$ and $\Theta=k x$, where

$$
R_{0}=\sqrt{c\left(\tau_{1 c}-\tau_{1}\right)-\tau_{1} k^{2}}
$$

and $k<\sqrt{c\left(\tau_{1 c}-\tau_{1}\right) / \tau_{1}}$. It represent the nonlinear dispersionless traveling waves of the phase of the domain oscillations with the wave vector $k$ which are stable if $k<\sqrt{c\left(\tau_{1 c}-\tau_{1}\right) / 3 \tau_{1}}$. A particular solution with $k=0$ corresponds to the synchronous oscillations of the domains. In fact, if the boundary conditions for the original reaction-diffusion system (3) and (44) are neutral, as in most of the real situations, this is the only admissible plane wave solution. Since it is stable, a variety of initial conditions will evolve into it, causing the oscillations of different domains to synchronize. This means that the synchronization of oscillations of different domains is the major scenario of the development of the small-period patterns in one dimension. Equations 36 and (37) also admit a solution in the form of a domain boundary (kink), upon going through which the phase $\Theta$ changes by $\pi$. These are the only stable stationary solutions of Eqs. (36) and (37), so any initial condition will in general produce a collection of regions in which the domains oscillate in phase. In the regions next to each other the domains will oscillate in antiphase. At $\tau_{1}<\tau_{1 c}$ Eqs. (36) and (37) also have the solutions in the form of the wave in which the stable state $R=R_{0}$ with $k=0$ and $\Theta=$ const invades the unstable state $R=0$. This wave corresponds to the onset of the synchronous oscillations in the unstable stationary strata from a localized initial condition. The propagation velocity of this wave is $v^{*}=\sqrt{c\left(\tau_{1 c}-\tau_{1}\right) / \tau_{1}}$ 30].

It is clear that small local inhomogeneities, both intrinsic and extrinsic, may play the role of the organizing centers for the one-dimensional analog of the target patterns: waves traveling away from the inhomogeneity in both directions. An example of an intrinsic inhomogeneity here is a non-uniform distribution of the domains along the $x$-axis. In this case instead of the constant density of domains we have a smooth function $n(x)$, which can also vary in time and can be considered as an extra degree of freedom in the problem. The density must satisfy the continuity equations

$$
\frac{\partial n}{\partial t}+\frac{\partial}{\partial x}(n \mathrm{v})=0
$$

where

$$
\mathrm{v}=\frac{\bar{\rho}}{\tau_{2}} \frac{\partial \bar{\eta}}{\partial x}
$$

is the velocity of the domain as a whole due to the gradient of $\bar{\eta}$ [see Eq. (15)], and

$$
\tau_{2}=\alpha Z / \epsilon B \mathcal{L}_{p}^{3}
$$

is the characteristic time scale of the density variation. For $\tau_{1} \sim 1$ we have $\tau_{2} \sim \mathcal{L}_{p}^{-2}$, so the density relaxes on much longer time scale and any non-uniform distribution of $n$ is frozen on the time scale of the relaxation of the amplitude and phase. This means that by setting a nonuniform density of domains as the initial condition one can produce complex spatio-temporal patterns of the domain oscillations which will not synchronize for a very long time.

From the definition of $R$ follows that it lies in the interval from $\bar{\rho}_{0}$ to $\frac{1}{2}-\bar{\rho}_{0}$. If $R$ exceeds one of these values, the domains will either merge or collapse in the course of the oscillations. On the other hand, according to Eq. (38), the value of $R_{0}$ may exceed either $\bar{\rho}_{0}$ or $\frac{1}{2}-\bar{\rho}_{0}$ for some values of $\tau_{1}$. This can be seen from Fig. 3, in which the bottom solid line corresponds to the values of $\tau_{1}$ at which this happen. Figure 3 thus shows that the synchronization of the domain oscillations occurs only in the relatively narrow region of the system's parameters. The merging or collapse of the domains will result in the breakdown of the collective domain oscillations and collapse of the strata. Indeed, suppose that at some moment every two domains merged into one. This may be viewed as doubling of the pattern's period $\mathcal{L}_{p}$. According to Eq. (28), this will result in the decrease of $\tau_{1}$ and further increase of $R_{0}$. Therefore, the process of domain merging will have an avalanche character and lead to a complete destruction of the strata. The way the breakdown of the strata will follow will depend on whether or not the homogeneous state of the systems is stable with respect to 
the uniform self-oscillations of the homogeneous state at this value of $A$. If the homogeneous state of the system is unstable, the breakdown will result in the onset of the uniform relaxation self-oscillations (the latter follows from the fact that since $\tau_{1 c} \lesssim 1$ and $\epsilon \ll 1$ we have $\alpha \ll 1$ and, therefore, relaxation oscillations [8 10]). If the homogeneous state of the system is stable, the breakdown will result in the formation of traveling domain patterns (AS) or in the collapse of the strata into the homogeneous state.

The scenarios of the evolution of one-dimensional strata of small period discussed in the previous paragraph are precisely what we see in the numerical simulations of a concrete model. We also find a detailed quantitative agreement between the limit cycle oscillations of a single domain determined by Eqs. (29) and (30) and the results of the numerical simulations of a reaction-diffusion model for small enough $\epsilon$ and $\mathcal{L}_{p}$.

Before concluding this section, let us discuss another dynamic behavior of strata in one dimension. As was recently shown by Osipov, for $\alpha \lesssim \epsilon$ periodic patterns may undergo the instability leading to the formation of the traveling strata (a wave train) 31. Osipov obtained the general criterion for this instability. In the case of one-dimensional strata of small period it is possible to calculate the threshold value of $\alpha$, since the spatial variation of $\eta$ is given by $\hat{\eta}(x)$. Substituting it into the criterion (Eq. (4.5) of Ref. [31]), we obtain that the transformation of the stationary strata to traveling occurs at $\alpha_{T} \sim \epsilon \mathcal{L}_{p}^{3}$ One can see that this instability occurs for much smaller values of $\alpha$ than the oscillatory instability for which $\alpha_{c} \sim \epsilon \mathcal{L}_{p}$. Therefore, the instability leading to the formation of the traveling pattern can never be reached for the one-dimensional strata of small period.

Throughout the analysis presented above we used the fact that the period of the pattern is small compared to the length of the variation of the inhibitor and used $\mathcal{L}_{p}$ as the small parameter in the expansions. The smallness of the parameter $\mathcal{L}_{p}$ greatly simplified the treatment of the domain interactions and lead to the universal results which are practically independent of the concrete nonlinearities of the system. This is a general property of the $\mathrm{KN}$ and $\mathrm{K} \Omega \mathrm{N}$ systems [15, 16,26]. Unfortunately, no such treatment is possible for the one-dimensional periodic patterns whose period is of order 1 . In the latter case one is faced with the problem of treating the dynamics of each individual domain separately and taking into account the complex memory effects associated with the domain interactions, which cannot be solved in general. So, the problem of the interaction and dynamics of the one-dimensional patterns consisting of the domains whose size if of order 1 remains open. This problem, however, does not exist in higher dimensions, if $\epsilon$ is small enough. In this case the domain sizes are necessarily small since the domains whose size is greater than $\epsilon^{1 / 3}$ are always unstable with respect to the transverse instability of their walls and split into smaller domains [15,26].

\section{HIGHER-DIMENSIONAL MULTIDOMAIN PATTERNS}

Let us now study periodic multidomain patterns in higher dimensions. For definiteness we will consider a two-dimensional hexagonal pattern of disk-shaped domains, the most frequent Turing pattern in chemical experiments [5]. As was shown by Muratov and Osipov, in two dimensions a stable stationary multidomain pattern must consist of the domains whose size is of order $\epsilon^{1 / 3}$ [15,16. Since the period of the pattern is of the same order as the domain size, it can be used as a natural small parameter, so all the ideas of the analysis of the previous section should apply to the two-dimensional multidomain patterns.

Let us proceed with the derivation of the equations describing the collective oscillations of the domains in the pattern. For a hexagonal pattern with the period $\mathcal{L}_{p}$ the density of the domains is $n=2 / \sqrt{3} \mathcal{L}_{p}^{2}$, so Eq. (18) becomes

$$
\frac{\partial \bar{\eta}}{\partial t}=\Delta \bar{\eta}-c_{1} \bar{\eta}\left(1+\frac{2 \pi c_{2} \rho^{2}}{\sqrt{3} \mathcal{L}_{p}^{2}}\right)-\frac{2 \pi a\left(\rho^{2}-\rho_{0}^{2}\right)}{\sqrt{3} \mathcal{L}_{p}^{2}}
$$

where we introduced

$$
\rho_{0}^{2}=-\frac{\sqrt{3} \mathcal{L}_{p}^{2} Q\left(\theta_{s 1}, \eta_{s}\right)}{2 \pi a} .
$$

Because of the anisotropy of the hexagonal lattice the shape of the domains will in general slightly deviate from the disk shape. It is clear that this deviation is small and is smaller when the radius of the domains is smaller. So, for the sake of simplicity and without significantly affecting the results of the analysis, we will ignore the effects of the anisotropy and consider the domains to be ideally circular. Also, instead of considering the problem for $\hat{\eta}$ in the hexagonal cell, we will solve it for a circular domain of radius $\mathcal{L}_{p} / 2$. Thus, we have

$$
\frac{d^{2} \hat{\eta}}{d r^{2}}+\frac{1}{r} \frac{d \hat{\eta}}{d r}=a I(r)-\frac{4 a \rho^{2}}{\mathcal{L}_{p}^{2}}, \quad\langle\hat{\eta}\rangle=0
$$

where $r$ is the radial coordinate and the boundary conditions are neutral at $r=0$ and $r=\mathcal{L}_{p} / 2$. Solving this equation for $\hat{\eta}$ and calculating $\hat{\eta}_{s}=\hat{\eta}(\rho)$, we get

$$
\hat{\eta}_{s}=a\left(\frac{3 \rho^{2}}{8}-\frac{3 \rho^{4}}{2 \mathcal{L}_{p}^{2}}+\frac{\rho^{2}}{2} \ln \frac{2 \rho}{\mathcal{L}_{p}}\right),
$$

so, Eq. 22) becomes

$$
\frac{\partial \rho}{\partial t}=-\frac{\epsilon^{2}}{\alpha \rho}+\frac{\epsilon B}{\alpha Z a}\left(\bar{\eta}+\frac{3 \rho^{2}}{8}-\frac{3 \rho^{4}}{2 \mathcal{L}_{p}^{2}}+\frac{\rho^{2}}{2} \ln \frac{2 \rho}{\mathcal{L}_{p}}\right) .
$$

Rescaling the variables according to Eq. (27) and dropping the primes, from Eqs. (42) and (46) we get 


$$
\begin{gathered}
\frac{\partial \bar{\eta}}{\partial t}=\Delta \bar{\eta}-c_{1} \bar{\eta}\left(1+\frac{2 \pi c_{2}}{\sqrt{3}} \bar{\rho}^{2}\right)-\frac{\pi}{\sqrt{3}} \omega_{0}^{2} \tau_{1}\left(\bar{\rho}^{2}-\bar{\rho}_{0}^{2}\right) \\
\tau_{1} \frac{\partial \bar{\rho}}{\partial t}=\bar{\eta}-\frac{\bar{\epsilon}}{\bar{\rho}}+\frac{3 \bar{\rho}^{2}}{8}-\frac{3 \bar{\rho}^{4}}{2}+\frac{\bar{\rho}^{2}}{2} \ln 2 \bar{\rho}
\end{gathered}
$$

where

$$
\bar{\epsilon}=\epsilon Z / B \mathcal{L}_{p}^{3}
$$

As before, $0<\bar{\rho}_{0}<\frac{1}{2}$. The parameter $\bar{\epsilon}$ does not appear in the one-dimensional case. It characterizes the stability of the domains with respect to the transverse perturbations. Since the curvature radius of the stable domains must be of order $\epsilon^{1 / 3}$ [15, 116], the value of $\bar{\epsilon}$ is of order 1 for the domains whose size is comparable with $\mathcal{L}_{p} \sim \epsilon^{1 / 3}$. Notice that Eqs. (47) and (48) without the space dependence and with the coefficient $\pi / \sqrt{3}$ replaced by 2 in Eq. (47) will describe the oscillations of a single domain in a circular region of radius $\mathcal{L}_{p} \ll 1$. This situation was realized in the experiment by Haim et al. on the ferrocyanide-iodate-sulfite (FIS) reaction [22].

When the condition of Eq. (31) is satisfied, Eqs. (47) and (48) have two time scales, as in the one-dimensional case: the short time scale $\omega_{0}^{-1}$ for the oscillations and the long time scale $\tau_{1}$ for the relaxation of their amplitude and phase. However, in contrast to the one-dimensional case, the analysis is complicated by the fact that now the oscillations themselves are nonlinear. To proceed further, we will use Eq. (48) to express $\bar{\eta}$ in terms of $\bar{\rho}$ and substitute it into Eq. (47), taking into account that $\omega_{0} \gg 1$. We obtain

$$
\frac{\partial^{2} \bar{\rho}}{\partial t^{2}}+\frac{\pi}{\sqrt{3}} \omega_{0}^{2}\left(\bar{\rho}^{2}-\bar{\rho}_{0}^{2}\right)=-\left(\frac{f(\bar{\rho})}{\tau_{1}}-\Delta\right) \frac{\partial \bar{\rho}}{\partial t},
$$

where

$$
\begin{aligned}
f(\bar{\rho})= & c_{1} \tau_{1}\left(1+\frac{2 \pi c_{2}}{\sqrt{3}} \bar{\rho}^{2}\right) \\
& -\frac{\bar{\epsilon}}{\bar{\rho}^{2}}-\frac{5 \bar{\rho}}{4}+6 \bar{\rho}^{3}-\bar{\rho} \ln 2 \bar{\rho} .
\end{aligned}
$$

The left-hand side of Eq. (50) is an equation of motion for a particle of unit mass in the potential $V=$ $\frac{\pi \omega_{0}^{2}}{\sqrt{3}}\left(\frac{\bar{\rho}^{3}}{3}-\bar{\rho}_{0}^{2} \bar{\rho}\right)$. For $0<\bar{\rho}<\frac{1}{2}$ it describes finite motion between $\bar{\rho}=\bar{\rho}_{\min }$ and $\bar{\rho}=\bar{\rho}_{\max }$ with the characteristic frequency $\omega_{0}$. The right-hand side of Eq. (50) is a weak nonlinear friction force which also contains the diffusion term that couples the oscillators in a spatially distributed system.

As in the case of the one-dimensional patterns, the equilibrium solution $\bar{\rho}=\bar{\rho}_{0}$ of Eq. (50) corresponds to the stationary multidomain pattern. Equation (50) shows that the stationary multidomain pattern becomes unstable (the friction $f$ becomes negative) with respect to the fluctuation with $\omega \cong \omega_{0}$ and $k=0$ when $\tau_{1}<\tau_{1 c}$, where

$$
\tau_{1 c}=\frac{4 \bar{\epsilon}+5 \bar{\rho}_{0}^{3}-24 \bar{\rho}_{0}^{5}+4 \bar{\rho}_{0}^{3} \ln 2 \bar{\rho}_{0}}{4 c_{1} \bar{\rho}_{0}^{2}\left(1+\frac{2 \pi c_{2}}{\sqrt{3}} \bar{\rho}_{0}^{2}\right)} .
$$

Going back to the original variables, we see that in the case of the two-dimensional multidomain pattern the destabilization occurs at

$$
\alpha_{c} \sim \epsilon^{4 / 3} .
$$

Note that the value of $\alpha_{c}$ is smaller than $\alpha_{\omega} \sim \epsilon$ at which a localized domain (AS) is destabilized with respect to pulsations [8,9,15].

Figure 4 shows the possible forms of the dependences $\tau_{1 c}\left(\bar{\rho}_{0}\right)$ obtained from Eq. (52) for different values of $\bar{\epsilon}$. One can see from Eq. (52) that for $\bar{\epsilon}>0.031$ for any value of $\bar{\rho}_{0}$ there exists a value of $\tau_{1}$ at which the instability occurs. When $0.00045<\bar{\epsilon}<0.031$ the instability is realized only for $\bar{\rho}_{0}<\bar{\rho}_{a 3}$ with $\bar{\rho}_{a 3}<\frac{1}{2}$, whereas for $\bar{\epsilon}<0.00045$ the instability is realized for $\bar{\rho}_{0}<\bar{\rho}_{a 1}$ and

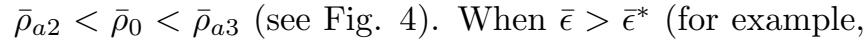
$\bar{\epsilon}^{*}=0.0034$ for $c_{2}=0$ ) for a given $\tau_{1}$ the instability is realized for a single value of $\bar{\rho}_{0}$, whereas for $\bar{\epsilon}<\bar{\epsilon}^{*}$ there are values of $\tau_{1}$ for which the instability is realized for three values of $\bar{\rho}_{0}$.

Let us take a closer look at Eq. (50). The behavior of the oscillations is determined by the sign of the friction term $f$ in Eq. (50). Three possible forms of this term as a function of $\bar{\rho}$ are shown in Fig. 5. The oscillation amplitude decreases if $\bar{\rho}$ remains in the domain where $f>0$, or increases if $\bar{\rho}$ is in the domain where

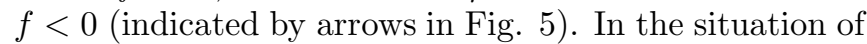
Fig. 5(c) all values of $\bar{\rho}$ correspond to the amplification region, so the amplitude of any oscillations will increase until $\bar{\rho}_{\text {max }}$ becomes equal to $\frac{1}{2}$ or until $\bar{\rho}_{\text {min }}=0$. In the first case the neighboring domains will merge while in the second the domain will collapse. Both these effects will cause the restructuring of the pattern and significant changes in the collective domain oscillations. In the situation of Fig. 5(b) the oscillations are amplified when $\bar{\rho}<\bar{\rho}_{c 3}$ and attenuated when $\bar{\rho}>\bar{\rho}_{c 3}$. The stationary multidomain pattern will be stable when $\bar{\rho}_{0}>\bar{\rho}_{c 3}$ or unstable when $\bar{\rho}_{0}<\bar{\rho}_{c 3}$. Depending on the signs of the first and the second derivatives of $f(\bar{\rho})$ the bifurcation at $\bar{\rho}_{0}=\bar{\rho}_{c 3}$ may be both supercritical and subcritical (this is a Hopf bifurcation). It is clear, however, that deeper into the unstable regime the oscillations will be amplified until they collapse when $\bar{\rho}_{\text {min }}=0$ even in the case of the supercritical bifurcation.

The most interesting situation is shown in Fig. E(a). There the oscillations are amplified when $\bar{\rho}<\bar{\rho}_{c 1}$ or $\bar{\rho}_{c 2}<\bar{\rho}<\bar{\rho}_{c 3}$ and attenuated for the rest of the values of $\bar{\rho}$, so the pattern is stable only for $\bar{\rho}_{c 1}<\bar{\rho}_{0}<\bar{\rho}_{c 2}$ or $\bar{\rho}_{0}>\bar{\rho}_{c 3}$. The bifurcation at $\bar{\rho}_{c 3}$ is supercritical, while the bifurcation at $\bar{\rho}_{0}=\bar{\rho}_{c 1}$ is subcritical. This can be easily seen from the following argument. By varying the 
value of $\tau_{1}$ one can make the minimum of $f$ between $\bar{\rho}_{c 2}$ and $\bar{\rho}_{c 3}$ arbitrarily shallow, thus controlling the amplification of the oscillations in the region $\bar{\rho}_{c 2}<\bar{\rho}<\bar{\rho}_{c 3}$. Since in this case the small region of amplification is surrounded by a finite region of dissipation, the bifurcation at $\bar{\rho}_{c 2}$ and $\bar{\rho}_{c 3}$ will be supercritical. Since the first and the second derivatives of $f$ at $\bar{\rho}_{c 1}$ are opposite to the one at $\bar{\rho}_{c 2}$, the bifurcation at $\bar{\rho}_{c 1}$ is subcritical. If the value of $\tau_{1}$ is decreased, the minimum of $f$ between $\bar{\rho}_{c 2}$ and $\bar{\rho}_{c 3}$ gets deeper, and at some value of $\tau_{1}$ the bifurcation at $\bar{\rho}_{c 2}$ becomes subcritical. Upon further decreasing the value of $\tau_{1}$ the limit cycle associated with the subcritical bifurcation at $\bar{\rho}_{c 2}$ will become unstable and lead to the collapse of the oscillations. Thus, the most stable limit cycle oscillations should be expected when $\bar{\rho}_{c 2}<\bar{\rho}_{0}<\bar{\rho}_{c 3}$ and the minimum of $f$ between $\bar{\rho}_{c 2}$ and $\bar{\rho}_{c 3}$ is sufficiently shallow. The analysis of the bifurcation types for different values of $\bar{\epsilon}$ and $\bar{\rho}_{0}$ is summarized in Fig. 6 (for simplicity we used $c_{2}=0$ ).

Up to now we discussed the oscillations of a single domain in the multidomain pattern. What we found is that in certain situations the synchronous oscillations of all domains in the pattern can be stable. This naturally implies that the synchronously pulsating multidomain pattern is an attractor and the synchronization of the domain oscillations is therefore one of the major scenarios of the development of the stationary multidomain patterns. However, the situation is reacher in two dimensions because of the possibility of other classes of stable solutions, such as target patterns and spiral waves. In principle, it is possible to use the fact that $\omega_{0}^{-1} \ll \tau_{1}$ and obtain the equations for the amplitude and phase by averaging over the trajectories of the frictionless oscillators in this case as well. This calculation however, cannot be carried out in the analytical form. On the other hand, in a wide region of the parameters the bifurcation of the stationary pattern is supercritical, so in its vicinity the dynamics of the pattern is described by the complex Landau-Ginzburg equation. In the appropriately scaled time and space variables this equation in the considered case has the form

$$
\frac{\partial W}{\partial t}=\Delta W+W-(1+i b)|W|^{2} W,
$$

where $W$ is appropriately normalized complex amplitude and

$$
b=\frac{4 \cdot 2^{1 / 2} \cdot 3^{3 / 4} \pi^{1 / 2} \bar{\rho}_{0}^{5 / 2} \omega_{0} \tau_{1 c}}{\frac{16 \pi}{\sqrt{3}} c_{1} c_{2} \tau_{1 c} \bar{\rho}_{0}^{4}-56 \bar{\epsilon}+216 \bar{\rho}_{0}^{5}+\bar{\rho}_{0}^{3}+4 \bar{\rho}_{0}^{3} \ln 2 \bar{\rho}_{0}} .
$$

The complex Landau-Ginzburg equation has been the subject of intensive studies for the last two decades (see, for example, Refs. [2, 27, 32,33]). Specifically, for the case of Eq. (54) Hagan obtained the solution in the form of a steadily rotating spiral wave [34]. In our case this spiral wave will be seen on top of the stationary multidomain pattern. Precisely this phenomenon was observed by Boissande, Dulos, and De Kepper in the experiments with the Turing patterns in the chlorite-iodide-malonic acid (CIMA) reaction 12.

Kuramoto and Koga showed that the spiral waves in Eq. (54) become unstable at sufficiently large $b$ [35]. They showed that the spiral breakup leads to the formation of a chaotic spatio-temporal pattern — spiral turbulence. This will be the case in the situation we study. Indeed, throughout the analysis we assumed that $\omega_{0} \tau_{1} \gg 1$, what means that $b \gg 1$, so in general the spiral wave solution is unstable. Note, however, that the value of $b$ decreases as $\mathcal{L}_{p}$ increases, so when $\mathcal{L}_{p}$ (and $\epsilon$ ) is not very small, the value of $b$ may become small enough, so that the spiral wave solution is stable. This can also be achieved by making the coupling constant $B$ smaller.

Since generally $b \gg 1$, plane wave solutions will be stable only in a narrow range of the wave vectors around $k=0$ [27. This is because, in contrast to the onedimensional case, the dispersion of the plane waves is high. However, for Eq. (54) the Benjamin-Feir instability is not realized, so the synchronous oscillations of the domains are always stable in the spatially distributed system close to the bifurcation point.

The stationary multidomain patterns are never perfect. The defects of the pattern will work as initiators for the spiral waves, which will in turn break up and produce more spiral waves invading the entire pattern, so the formation of the chaotically oscillating multidomain pattern is the more likely scenario for the development of the unstable stationary multidomain pattern in two dimensions.

The imperfections of the multidomain pattern may also work as the guiding centers generating target waves of the oscillation phase 27]. An argument similar to the one applied to the one-dimensional strata may be applied here. The target waves may be initiated by the smooth spatial variations of the density of domains. The equation for the density will be given by Eqs. (39) and (40), if one replaces $\partial / \partial x$ by $\nabla$, so the time scale of the density variation will once again be much longer than $\tau_{1}$, and, therefore, the density can be considered frozen on the latter time scale. However, since the dispersion of the waves in the considered situation is high, the target patterns are likely to be unstable, what should also lead to the stochastization of the domain oscillations.

It is clear that qualitatively the scenarios discussed above are also realized when $\tau_{1}$ is not very close to $\tau_{1 c}$. Of course, for arbitrary $\tau_{1}$ one should also take into account the possibility of the collapse or merging of the domains in the course of the oscillations. To determine the values of $\tau_{1}$ at which the collapse or merging of the domains occur for a given value of $\bar{\rho}_{0}$ one can consider the oscillator in Eq. (50) without the space dependence. The collapse or merging of the domains occurs when $\bar{\rho}_{\min }=0$ or $\bar{\rho}_{\max }=\frac{1}{2}$, respectively. The values of $\bar{\rho}_{\min }$ and $\bar{\rho}_{\max }$ can be obtained from the condition of energy balance for the steady oscillations. Recalling that the friction is weak, from the elementary mechanics we get 


$$
\int_{\bar{\rho}_{\text {min }}}^{\bar{\rho}_{\max }} f(\bar{\rho}) \sqrt{2\left(E-\frac{\pi \omega_{0}^{2}}{\sqrt{3}}\left(\frac{\bar{\rho}^{3}}{3}-\bar{\rho}_{0}^{2} \bar{\rho}\right)\right)}=0,
$$

where $E$ is the energy of the oscillator corresponding to the values of $\bar{\rho}_{\min }$ and $\bar{\rho}_{\max }$. We solved this equation numerically in the case $c_{2}=0$. Figure 7 shows a typical dependence of the amplitude of the oscillations on $\tau_{1}$ for a given value of $\bar{\epsilon}$ in the case of the supercritical bifurcation $\left(\bar{\rho}_{\text {min }}\right.$ and $\bar{\rho}_{\text {max }}$ correspond to the left and the right portions of the solid curve, respectively). From this figure it can be seen that the limit cycle oscillations are stable only if $\tau_{1 d}<\tau_{1}<\tau_{1 c}$. If $\tau_{1}<\tau_{1 d}$, the amplitude of the oscillations will increase until the domain collapses or merges with the neighbors. Several possibilities exist for the values of $\bar{\rho}_{\min }^{d}$ and $\bar{\rho}_{\max }^{d}$. The value of $\tau_{1 d}$ may happen to be negative, in this case the stability of the limit cycle oscillations will depend on whether the value of $\bar{\rho}_{\text {max }}$ becomes greater than $\frac{1}{2}$ at some value of $\tau_{1}$. The oscillations will be stable for $\tau_{1}$ greater than this value if this is the case, or stable for all values of $\tau_{1}$ in the opposite case. The value of $\bar{\rho}_{\text {max }}^{d}$ may also happen to be greater than $\frac{1}{2}$. In this case the region of the stability of the domain oscillations will also be narrower. Notice that $\bar{\rho}_{\text {min }}^{d}$ is always greater than zero because of the singular character of $f(\bar{\rho})$ at $\bar{\rho}=0$.

In the case of subcritical bifurcation the limit cycle oscillations are unstable for any values of $\tau_{1}$, so collective oscillations of the domains in the multidomain pattern always break down in this case. The most likely scenario here is that some of the domains will shrink and collapse whereas some will grow, what can be effectively thought of as an increase of the pattern's period. As a result, the value of $\bar{\epsilon}$, which mainly determines the type of the bifurcation, will get smaller, so in the new pattern of greater period the bifurcation may happen to be supercritical. This means that the pattern may naturally evolve into a state in which the collective domain oscillations are stable from more or less arbitrary initial conditions, provided that the initial condition consists of the alternating hot and cold domains of size less or of or$\operatorname{der} \epsilon^{1 / 3}$. It is also possible that the pattern that forms in this process is stable with respect to the domain oscillations. Yet another possibility is that in this process some of the domains will merge, what will result in the formations of an irregular pattern, which also may exhibit chaotic dynamics. The merging process may also lead to the destruction of the multidomain pattern. We will get back to these points when we will discuss the results of the numerical simulations of a concrete model.

In the analysis presented in this section we did not specify precisely the range of the values of $\bar{\epsilon}$. This range is determined by the stability of the stationary multidomain pattern with respect to the activator repumping and the transverse distortions of the walls of the domains. When $\bar{\epsilon}$ is greater than some value for a given $\bar{\rho}_{0}$, the pattern becomes unstable with respect to the activator repumping effect leading to the doubling of the pattern's period, whereas when $\bar{\epsilon}$ is smaller than some critical value at a given $\bar{\rho}_{0}$, the domains destabilize with respect to the transverse instability of their walls leading to the radially-nonsymmetric distortions of the domains [8,10,16,36]. Both these instabilities occur when $\bar{\epsilon} \sim 1$ in the limit $\epsilon \rightarrow 0$ regardless of the value of $\alpha$. It is possible to show [36] that for the stable stationary multidomain pattern in two dimensions the value of $\bar{\epsilon} \sim 0.002$, so the scenarios discussed above are indeed realized. Also, according to the Osipov's criterion [31], the transformation of a stationary multidomain pattern into traveling will occur when $\alpha_{T} \sim \epsilon^{2} \ll \alpha_{c}$.

For the purpose of completeness let us quote the equations obtained in the case of the three-dimensional multidomain pattern consisting of spherical domains situated on a closed-packing lattice. The derivation follows along the same lines as the derivation in two dimensions. Introducing

$$
\rho_{0}^{3}=-\frac{3 \mathcal{L}_{p}^{3} Q\left(\theta_{s 1}, \eta_{s}\right)}{4 \pi \sqrt{2} a},
$$

and rescaling $\bar{\eta}$ and $\rho$ according to Eq. (27), we obtain

$$
\frac{\partial \bar{\eta}}{\partial t}=\Delta \bar{\eta}-c_{1} \bar{\eta}\left(1+\frac{4 \pi \sqrt{2}}{3} c_{2} \bar{\rho}^{3}\right)-\frac{2 \pi \sqrt{2}}{3} \omega_{0}^{2} \tau_{1}\left(\bar{\rho}^{3}-\bar{\rho}_{0}^{3}\right),
$$

$$
\tau_{1} \frac{\partial \bar{\rho}}{\partial t}=\bar{\eta}-\frac{2 \bar{\epsilon}}{\bar{\rho}}-\frac{\bar{\rho}^{2}}{3}+\frac{6 \bar{\rho}^{3}}{5}-\frac{32 \bar{\rho}^{5}}{15}
$$

The properties of these equations are essentially the same as in two dimensions.

\section{ENTRAINMENT OF THE OSCILLATIONS IN A DISORDERED PATTERN}

The multidomain patterns that we studied so far consisted of domains situated on a regular lattice. We found that the motion of the domains occurs on two time scales: the short time scale $\omega_{0}^{-1}$ which is proportional to the period of the oscillations of a single domain and the long time scale $\tau_{1}$. The motion of the domains on the time scale $\omega_{0}^{-1}$ is conservative, so the time $\tau_{1}$ is associated with the relaxation of the oscillator's energy. In two dimensions we found a lot of the situations in which the collective domain oscillations should become chaotic, either because of the nonlinear dynamics of the oscillation amplitude and phase, or because of the defects. This chaotic dynamics, however, is realized on the long time scale and is essentially determined by the nonlinear relaxation processes. Here we would like to ask the following question. Suppose that instead of the domains of a fixed radius on a perfect lattice (hexagonal in two dimensions), we have a random arrangement of the domains with a distribution of radii. What will be the dynamics of different domains on the short time scale $\omega_{0}^{-1}$ ? 
To proceed, we will consider a simplified situation and ignore the space dependence of all dynamical quantities in the problem. One can think that the domains are considered in the system whose size is much smaller than 1 . Let $n(\rho, t)$ now be the distribution function of the domain radii $\rho$. The distribution $n(\rho, t)$ must satisfy the Liuville equation obtained from Eq. (22). Rescaling appropriately $n, \bar{\eta}, \rho$, and $t$, and neglecting the terms which are not significant on the time scale $\omega_{0}^{-1}$, from Eq. (18) and (22) we get

$$
\begin{gathered}
\frac{\partial \bar{\eta}}{\partial t}=\rho_{0}^{d}-\int \rho^{d} n(\rho, t) d \rho \\
\frac{\partial n}{\partial t}=\bar{\eta} \frac{\partial n}{\partial \rho}
\end{gathered}
$$

where $\rho_{0}^{d}$ is a constant which comes from the term $Q\left(\theta_{s 1}, \eta_{s}\right)$ in Eq. (18) and the distribution $n(\rho, t)$ is normalized to unity:

$$
\int n(\rho, t) d \rho=1
$$

The last condition implies the conservation of the number of the domains as a function of time. This condition is in fact not always satisfied, since we have an absorbing boundary condition for $n$ at $\rho=0$ and even more sophisticated situation at large $\rho$ because of the possibility of the domain merging. It is clear, however, that this condition will be satisfied if the distribution $n$ has finite support at all times and the possibility of merging is excluded.

Let us introduce the quantities

$$
m_{0}=1, \quad m_{k}(t)=\int \rho^{k} n(\rho, t) d \rho,
$$

where $k=1,2, \ldots, d$. Then from Eqs. (60) and 61) follows:

$$
\begin{gathered}
\frac{d m_{k}}{d t}=-k \bar{\eta} m_{k-1}, \\
\frac{d \bar{\eta}}{d t}=\rho_{0}^{d}-m_{d} .
\end{gathered}
$$

Equations (64) and (65) can be solved exactly. One can use Eq. (64) with $k=0$ to eliminate $\bar{\eta}$ from Eq. (64) with $k=1$ and integrate this equation to get $m_{2}=$ $m_{1}^{2}+C_{1}$, where $C_{1}$ is a constant of integration. This equation, together with Eq. (64) with $k=0$ can be used to integrate Eq. (64) with $k=2$ to get $m_{3}=$ $m_{1}^{3}+3 C_{1} m_{1}+C_{2}$, and so forth. Thus, the whole set of Eqs. 64 and (65) can be reduced to a single equation for $m_{1}$. The constants of integration are determined by the initial distribution function $n_{0}(\rho)$. If one knows the solution of the equation for $m_{1}$, one then gets

$$
n(\rho, t)=n_{0}\left(\rho-m_{1}(t)+m_{1}(0)\right)
$$

The first conclusion one draws from the solution of Eqs. (64) and (65) is that the oscillations of different domains in a random pattern are always entrained. This is in fact obvious right from the start, since the rate of change of the radii for each domain is proportional to $\bar{\eta}$ which in turn depends integrally on the distribution of radii. However, the dynamics of the domains is determined by the initial distribution $n_{0}$ and may be qualitatively different for different initial conditions, even if the parameters of the system are the same.

Let us see what happens when $d=1,2,3$. In the case $d=1$ the equation for $m_{1}$ is

$$
\frac{d^{2} m_{1}}{d t^{2}}+m_{1}-\rho_{0}=0
$$

One can see that for any distribution $n_{0}$ (with finite support and and assuming that no domain merging occurs) the motion is that of a simple harmonic oscillator. The energy of the oscillator is determined by the value of $\bar{\eta}$ at $t=0$. One can see that dynamics of the random pattern on the time scale $\omega_{0}^{-1}$ is identical to the that of the ideally periodic pattern.

In $d=2$ and $d=3$ the situation becomes somewhat more complicated. In $d=2$ we have

$$
\frac{d^{2} m_{1}}{d t^{2}}+m_{1}^{2}+C_{1}-\rho_{0}^{2}=0
$$

Here again the dynamics is equivalent to that of an ideal hexagonal pattern, but the equilibrium point is shifted. The shift is determined by $C_{1}=\left\langle\rho^{2}\right\rangle-\langle\rho\rangle^{2}$ at $t=0$. One can see from Eq. (68) that when $C_{1}>\rho_{0}^{d}$ the oscillations are not possible. In $d=3$ we have

$$
\frac{d^{2} m_{1}}{d t^{2}}+m_{1}^{3}+3 C_{1} m_{1}+C_{2}-\rho_{0}^{3}=0,
$$

and the dynamics of the domains may be different from that of the domains in an ordered pattern.

The main conclusion that follows from the analysis above is that the disorder, especially small, should not significantly affect the dynamics of the pattern and that the equations for $\bar{\eta}$ and $\bar{\rho}$ should adequately describe the behavior of irregular multidomain patterns as well. It is also clear that the distortions of the domain shapes will not have significant effect either.

\section{SIMULATIONS OF A CONCRETE MODEL}

In this section we present the result of the numerical simulations of the model with a simple cubic nonlinearity:

$$
\begin{aligned}
& q=\theta^{3}-\theta-\eta, \\
& Q=\theta+\eta-A .
\end{aligned}
$$


Recently, Muratov and Osipov performed extensive numerical simulations of this model in two dimensions [16]. The emphasis of their work was on the instabilities of the localized solitary structures (AS) and the evolution of the patterns excited by a short localized external stimulus in the stable homogeneous system. They were able to construct a state diagram showing what kinds of patterns are realized for different values of $\alpha$ and $A$ for a fixed value of $\epsilon \ll 1$ from localized initial conditions. Here we perform numerical simulations of Eqs. (3) and (4) with (70) and (71) with non-localized initial condition.

The homogeneous state of the system under consideration is stable when $|A|>1 / 3 \sqrt{3} \simeq 0.19$. The various constants involved are 16,26

$$
a=2, \quad B=4, \quad Z=\frac{2 \sqrt{2}}{3}, \quad c_{1}=\frac{3}{2}, \quad c_{2}=0 .
$$

Unfortunately, the direct simulations of Eqs. (3) and (4) with $\epsilon \ll 1$ in the system of sufficiently large size are extremely time-consuming even on a very fast computer. In order for the equations for $\bar{\rho}$ and $\bar{\eta}$ to be in quantitative agreement with the simulations we should have $\epsilon \lesssim 0.01$. Nevertheless, it is expected to have qualitative agreement with the predictions of the preceding sections. All our simulations were performed with $\epsilon=0.05$.

Our first observation is that if a stable stationary multidomain pattern is taken as an initial condition in the run with sufficiently small $\alpha$, the pattern will destabilize with respect to the synchronous oscillations of the domains, and its evolution will depend on how small the value of $\alpha$ is and on whether the homogeneous state of the system is stable. When $\alpha$ is slightly below the threshold value and the pattern's period is not very small, the oscillations of different domains will synchronize. If the value of $\alpha$ is smaller, the domains may start to merge during the oscillations, what will result in the formation of an irregular pulsating pattern. If $\alpha$ is even smaller and the homogeneous state of the system is stable, the pattern will collapse into the homogeneous state after a few periods of oscillations or transform into a turbulent pattern. The turbulence here is induced by the selfreplication of the domains and is qualitatively different from the chaotic behaviors discussed above [16]. If the value of $\alpha$ is small enough and the homogeneous state of the system is unstable with respect to the uniform selfoscillations, the multidomain pattern will collapse into the uniform self-oscillations. This is in agreement with the predictions of our analysis.

Besides the synchronous oscillations of the domains, we predicted traveling waves of the oscillation phase. These traveling waves are indeed realized in the simulations (Fig. 8). One has to use the special initial conditions to excite a traveling wave. The distributions of $\theta$ should consist of the domains whose radii vary smoothly along the $x$-axis, and the distribution of $\eta$ should follow this variation with the phase difference of $\pi / 2$.

In addition to the plane waves, target waves of the oscillation phase are observed. Figure 9 shows a portion of a target wave emanating from the top left corner of the system. The wave breaks down after a while, as the domains begin to merge during the oscillations. As a result, an irregular chaotically oscillating pattern forms in the system. Notice that eventually, when no merging occur any longer, the average size of the domains becomes greater than the size of the domains at the beginning. This can be interpreted as a self-organized increase of the patterns period leading to the stabilization of the pattern's oscillations.

In another simulation in which the value of $\alpha$ was smaller, the target pattern broke down not only because of merging, but also because of the collapse of smaller domains (Fig. 10). As a result of the domain collapse, patches of the almost homogeneous state formed in the system. These patches are then invaded by the domains, what leads to the formation of an irregular pulsating pattern. For smaller values of $\alpha$ this may also lead to the formation of autowaves and turbulence induced by selfreplication of domains [16].

In the simulations above we used a hexagonal pattern as the initial condition. Let us now see what happens in the more realistic situation when the multidomain pattern is disordered. Figure 11 shows the evolution of such pattern. The initial stationary pattern was in fact generated as a result of the Turing instability of the homogeneous state at large $\alpha$. In the simulation of this figure the value of $\alpha$ is small enough, so that the stationary multidomain pattern is unstable, and a small disturbance was added to $\eta$ in the vicinity of the top left corner of the system in order to make the amplitude of the oscillations in this region bigger. We see that at early times (the first three plates in Fig. 11) the oscillations of the domains are indeed entrained, despite the differences in the domain sizes and shapes. Notice that in this simulation the homogeneous state of the system is unstable with respect to the uniform self-oscillations. One can see that here the amplitude of the oscillations increases until merging and collapse of the domains occur at the top left corner, which are then followed by the formation of the uniform self-oscillations. The uniform self-oscillations eventually invade the whole system and destroy the multidomain pattern. Yet the uniform self-oscillations and the multidomain pattern may coexist for quite a long time. This effect was observed in the dynamics of the Turing pattern in the CIMA reaction 12.

Above we considered the dynamics of the patterns consisting of circular hot domains in the cold system. Clearly, qualitatively the same dynamics is expected for the cold domains in the hot system. In fact, by changing $\theta \rightarrow-\theta, \eta \rightarrow-\eta$, and $A \rightarrow-A$ in the concrete system under consideration we will transform hot domains into cold and vice versa. A possibility exists for such oscillations of the multidomain pattern in which the hot domains will transform into cold domains and back. This process is illustrated in Fig. 12. There a stationary multidomain pattern which formed as a result of the Turing instability at $A=-0.1$ when the hot domains are favor- 
able was taken as the initial condition. The simulation was performed at $A=0.1$ when the cold domains are favorable. One can see the sequence of transitions from the hot domains to cold and back during the oscillations. A lot of the domains merge as their size increases, so the multidomain pattern quickly transforms into an irregular pattern consisting of many disconnected pieces. The connectivity of the domains changes with time. After $t=1.4$ the systems eneters a periodic cycle in which the pattern changes periodically from hot to cold. In the run with these parameters the pattern eventually collapsed into the homogeneous self-oscillations.

So, in summary, the collapse and merging of the domains appear to be the major cause for the chaotization of the oscillations. The chaos here is due to the underlying irregularity of the domains themselves. The predicted chaotic behavior near the onset of synchronous domain oscillations seems to be beyond the capabilities of the available computational power. While we were able to see steady non-uniform oscillations of a hexagonal multidomain pattern of period $\mathcal{L}_{p}=2.3$ at $\epsilon=0.1, \alpha=0.05$, and $A=-0.1$ in the system $40 \times 40$, the system was yet too small to identify these oscillations with chaos.

\section{CONCLUSION}

Thus, in this paper we studied the dynamics of the multidomain patterns of small period in $\mathrm{K} \Omega \mathrm{N}$ systems. In order to simplify the problem we took the advantage of the smallness of $\epsilon$, the natural small parameter in the considered systems $[8-10,15]$. In the limit $\epsilon \rightarrow 0$ the dynamics of the pattern reduces to the free boundary problem [18,26]. This problem may be further simplified by using the smallness of the pattern's period. This approach is somewhat limited in one dimension, but is always applicable to higher-dimensional multidomain patterns, if $\epsilon$ is small enough. In the case of the periodic patterns we managed to reduce the number of dynamical variables involved to the average domain radius $\rho$ and the average value of $\eta$. The equations for these quantities turned out to be universal. The nonlinearities of the original reaction-diffusion system enter only via a few numerical constants of order 1 . In $d \geq 2$ the dynamics studied by us is in fact the asymptotic limit of the true dynamics as $\epsilon \rightarrow 0$. Note, however, that the value of $\epsilon$ has to be sufficiently small in order for this approach to be in quantitative agreement with the actual dynamics. For example, for the concrete model studied in Sec. VI this is the case only when $\epsilon \lesssim 0.01$ (see also Ref. [16]). Nevertheless, the qualitative agreement is good for $0.01 \lesssim \epsilon \ll 1$.

The analysis performed by us predicts both synchronization and chaos in the collective domain oscillations of the multidomain patterns. There are two types of chaos that are realized: the chaos associated with the effects of the interplay of the dissipation and dispersion of the nonlinear waves of the phase of the domain oscillations and the chaos associated with the irregularity of the underlying multidomain pattern. The first kind of chaos is shown to correspond to the intermittency and defect turbulence in the complex Landau-Ginzburg equation. The second kind of chaos is due to the breakdown of the averaged dynamics description and is associated with the collapse and merging of the domains and the destruction of the regular multidomain pattern. Nevertheless, it is shown that even in an irregular pattern the domain oscillations are synchronized locally, so the chaos is still realized on the time and length scales of the dissipation processes.

Recall that $\epsilon=\sqrt{\alpha D_{\theta} / D_{\eta}}$, where $D_{\theta}$ and $D_{\eta}$ are the diffusion coefficients of the activator and the inhibitor, respectively. According to its definition, the value of $\epsilon$ is small as long as $\alpha$ is small and $D_{\eta} \gtrsim D_{\theta}$. However, if the values of $D_{\theta}$ and $D_{\eta}$ are of the same order, as is the case in typical experiments with the autocatalytic reactions [37], this means that $\alpha \sim \epsilon^{2}$. At this relationship between $\alpha$ and $\epsilon$ the stationary patterns are always unstable [8 10,15,37, and only autowaves will be realized. Our analysis also shows that this will be the case for the multidomain patterns. In other words, one should have $D_{\eta} \gg D_{\theta}$ in order for the stationary and more complex dynamic patterns (not autowaves) to be feasible in an experiment. As was emphasized by Epstein and Lengyel, in chemical systems, for example, one has to devise special methods to make the ratio of the diffusion coefficients large 37. On the other hand, the value of $\alpha$ is determined by the kinetics of the chemical reactions involved and can be easily made small.

It is well known that a solitary pattern (AS) in one dimension would destabilize and transform into a pulsating pattern when $\alpha \sim \epsilon$, what implies that $D_{\theta} / D_{\eta} \sim \alpha$ [8 10, 15, 17, 38]. The same condition must be satisfied in order for the instability of the homogeneous state of the system with respect to the uniform self-oscillations be before the Turing instability of the homogeneous state 8 10,16. This is a rather strict requirement for $\alpha \ll 1$. On the other hand, as was shown in Sec. IV, the destabilization of the stationary multidomain patterns in higher dimensions occurs when $\alpha \sim \epsilon^{4 / 3}$, what implies that in this case $D_{\theta} / D_{\eta} \sim \alpha^{1 / 2}$. This condition requires considerably smaller difference in the diffusion coefficients of the activator and the inhibitor.

Another interesting implication of our results is that in some situations it is possible that the oscillatory behavior of the "homogeneous" system is actually the consequence of the dynamics of the underlying multidomain pattern. Indeed, for the reasonable values of $D_{\eta} \sim 2 \times 10^{-5} \mathrm{~cm}^{2} \mathrm{~s}^{-1}$ and $\tau_{\eta} \sim 10 \mathrm{~s} 37$ we would have that the size of an individual domain is much smaller than $L \sim 1.5 \times 10^{-2} \mathrm{~cm}$, so the domains may actually lie beyond the resolution of the experiment. Notice that in the case $\epsilon \ll 1$ the oscillatory instability of the homogeneous state is always accompanied by the Turing instability [8 10]. Numerical simulations show that Turing patterns may form as a result of the instability of the homogeneous state even if the homogeneous state is unstable with respect to the uni- 
form oscillations and may in fact persist for even smaller $\alpha$ 16. So, we suggest that in certain situations it is the Turing patterns that exhibit the oscillatory behavior, whereas the system's bulk kinetics has relaxation character. If this is the case, one would expect to see the coexistence of the relaxation and oscillatory kinetics in the system, and multiplicity of the oscillation modes in a single system with the same parameters. The latter is the consequence of the fact that the characteristic time and length scales of the oscillations of the multidomain patterns strongly depend on the pattern's period which is not uniquely determined by the system's parameters.

The dynamics of the multidomain patterns discussed in the present paper was observed by Boissande, Dulos, and De Kepper in the experiments on CIMA reaction 12]. They were actually able to follow the dynamics of the domains and see synchronization, waves of the oscillation phase, including spirals on top of the multidomain pattern, merging of the domains and the coexistence of the domain oscillations and the uniform oscillations of the homogeneous state (the Hopf-holes, as they called them). They also emphasized that the waves they observed are essentially different from those in the Belousov-Zhabotinsky (BZ) reaction. In the CIMA reaction these are phase waves, as opposed to the autowaves in the BZ reaction. This is precisely the conclusion of our analysis.

Synchronously oscillating domains were also observed by Rose et al. in the experiments on the catalytic CO oxidation on the platinum surface [14]. The authors suggested that these oscillations may be explained by the introduction of global coupling. The results of the present paper suggest a more natural explanation for this phenomenon as dynamics of the multidomain pattern. To do this, one has to introduce a diffusion term into the equation for the inhibitor in the two-variable reactiondiffusion model of this reaction, which is otherwise known to have relaxation kinetics [39]. This would also seem to explain the transition from a target pattern into a cellular structure observed in these experiment. We would also like to mention the observation of synchronously pulsating cellular flames in the combustion experiments [13.

Haim et al. observed experimentally a single breathing domain in the FIS reaction in a circular reactor [22]. Their results agree with the conclusions of Sec. IV concerning the motion of a single domain. Indeed, they see the supercritical Hopf bifurcation from the stationary to the breathing domain, growing anharmonicity of the oscillations for larger amplitudes of the oscillations, and collapse of the domain for yet larger amplitudes. In this situation Eq. (50) (with $\pi / \sqrt{3}$ replaced by 2) might be used for the quantitative explanation of these effects.

The author would like to acknowledge the computational support from the Center for Computational Science at Boston University.
[1] G. Nicolis and I. Prigogine, Self-organization in NonEquilibrium Systems (Wiley Interscience, New York, 1977).

[2] M. Cross and P. C. Hohenberg, Rev. Mod. Phys. 65, 851 (1993).

[3] Oscillating and Traveling Waves in Chemical Systems, edited by R. J. Field and M. Burger (Wiley Interscience, New York, 1985).

[4] A. S. Mikhailov, Foundations of Synergetics (SpringerVerlag, Berlin, 1990).

[5] Chemical waves and patterns, edited by R. Kapral and K. Showalter (Kluwer, Dordrecht, 1995).

[6] J. D. Murray, Mathematical Biology (Springer-Verlag, Berlin, 1989).

[7] A. V. Gurevich and R. G. Mints, Rev. Mod. Phys. 59, 941 (1987).

[8] B. S. Kerner and V. V. Osipov, Autosolitons: a New Approach to Problems of Self-Organization and Turbulence (Kluwer, Dordrecht, 1994).

[9] B. S. Kerner and V. V. Osipov, Sov. Phys. - Uspekhi 32, 101 (1989).

[10] B. S. Kerner and V. V. Osipov, Sov. Phys. - Uspekhi 33, 679 (1990).

[11] Nonlinear dynamics and pattern formation in semiconductors and devices, edited by F. J. Niedernostheide (Springer, Berlin, 1994).

[12] J. Boissande, E. Dulos, and P. De Kepper, in Chemical waves and patterns, edited by R. Kapral and K. Showalter (Kluwer, Dordrecht, 1995). See Ref. [5].

[13] M. Gorman, M. el Hamdi, and K. A. Robbins, Combust. Sci. Tech. 98, 79 (1994).

[14] K. C. Rose et al., Phys. Rev. Lett. 76, 3582 (1996).

[15] C. B. Muratov and V. V. Osipov, Phys. Rev. E 53, 3101 (1996).

[16] C. B. Muratov and V. V. Osipov, Phys. Rev. E (to be published).

[17] S. Koga and Y. Kuramoto, Prog. Theor. Phys. 63, 106 (1980).

[18] T. Ohta, M. Mimura, and R. Kobayashi, Physica D 34, 115 (1989).

[19] A. Hagberg, E. Meron, I. Rubinstein, and B. Zaltzman, Phys. Rev. E (to be published).

[20] K. M. Mayer, J. Parisi, J. Peinke, and R. P. Huebner, Physica D 32, 306 (1988).

[21] V. P. Baev, A. V. Gurevich, R. G. Mints, and M. S. Ushomirskiy, Sov. Phys. - Solid State 24, 886 (1982).

[22] D. Haim et al., Phys. Rev. Lett. 77, 190 (1996).

[23] M. Gorman, M. el Hamdi, and K. A. Robbins, Combust. Sci. Tech. 98, 47 (1994).

[24] T. Ohta, A. Ito, and A. Tetsuka, Phys. Rev. A 42, 3225 (1990).

[25] T. Ohta and H. Nakazawa, Phys. Rev. A 45, 5504 (1992).

[26] C. B. Muratov, Phys. Rev. E (to be published).

[27] Y. Kuramoto, Chemical oscillations, waves, and turbulence (Springer-Verlag, New York, 1984).

[28] N. N. Bogoliubov and Y. A. Mitropolsky, Asymptotic methods in the theory of non-linear oscillations (Gordon and Breach Science Publishers, New York, 1961).

[29] A. C. Newell and J. A. Whitehead, J. Fluid Mech. 38, 279 (1969). 
[30] W. van Saarloos, Phys. Rev. A 37, 211 (1988).

[31] V. V. Osipov, Physica D 93, 143 (1996).

[32] W. van Saarloos and P. C. Hohenberg, Physica D 56, 303 (1992).

[33] H. Chaté, Nonlinearity 7, 185 (1994).

[34] P. Hagan, SIAM J. Appl. Math. 42, 762 (1982).

[35] Y. Kuramoto and S. Koga, Prog. Theor. Phys. 66, 1081 (1981).

[36] C. B. Muratov, (unpublished).

[37] I. R. Epstein and I. Lengyel, Physica D 84, 1 (1995).

[38] Y. Nishiura and M. Mimura, SIAM J. Appl. Math. 49, 481 (1989).

[39] M. Bär, N. Gottschalk, M. Eiswirth, and G. Ertl, J. Chem. Phys. 100, 1202 (1994).

FIG. 1. The qualitative form of the nullclines of Eqs. (11) and (2).

FIG. 2. The distributions of $\theta$ and $\eta$ in a stationary one-dimensional periodic strata.

FIG. 3. The parameter regions for oscillations and breakdown of the one-dimensional periodic strata. The upper solid line is the instability threshold for the multidomain pattern with respect to the oscillations. Below the bottom sold line the collective oscillations of the strata break down.

FIG. 4. The dependence $\tau_{1 c}\left(\bar{\rho}_{0}\right)$ from Eq. (52) for different values of $\bar{\epsilon}$.

FIG. 5. The nonlinear friction term $f(\bar{\rho})$ in Eq. (50): (a) $\bar{\epsilon}=0.00025, \tau_{1}=0.03$; (b) $\bar{\epsilon}=0.0075, \tau_{1}=0.175$; (c) $\bar{\epsilon}=0.05, \tau_{1}=0.025$. Other parameters are $c_{1}=1, c_{2}=0$.

FIG. 6. The bifurcation diagram for the two-dimensional stationary multidomain pattern for $c_{2}=0$. The numbers 1,2 , and 3 in the figure correspond to the bifurcations at $\bar{\rho}_{0}=\bar{\rho}_{c 1}$, $\bar{\rho}_{0}=\bar{\rho}_{c 2}$, and $\bar{\rho}_{0}=\bar{\rho}_{c 3}$, respectively. The shaded regions are those where the instability is not realized $\left(\tau_{1 c}<0\right)$.

FIG. 7. The sweep of the oscillations for given values of $\tau_{1}$ (horizontal lines below the solid curve), obtained from the solution of Eq. (56). The parameters used: $\bar{\epsilon}=0.00025$, $\bar{\rho}_{0}=0.25, c_{1}=1$, and $c_{2}=0$.

FIG. 8. The wave of the phase of the domain oscillations traveling from left to right. Distributions of the activator for different times. The parameters used are: $\epsilon=0.05, \alpha=0.02, A=-0.4$. The system is $20 \times 4$. The boundary conditions are periodic.
FIG. 9. Breakdown of a target wave of the oscillation phase. Distributions of the activator for different times. The parameters used are: $\epsilon=0.05, \alpha=0.019, A=-0.2$. The system is $20 \times 20$. The boundary conditions are periodic.

FIG. 10. Breakdown of a target wave of the oscillation phase for smaller $\alpha$. Distributions of the activator for different times. The parameters used are: $\epsilon=0.05, \alpha=0.015, A=-0.2$. The system is $20 \times 20$. The boundary conditions are neutral.

FIG. 11. Breakdown of the collective domain oscillations into the uniform self-oscillations. Distributions of the activator for different times. The parameters used are: $\epsilon=0.05, \alpha=0.015, A=-0.1$. The system is $20 \times 20$. The boundary conditions are neutral.

FIG. 12. The oscillations leading to the interconversion between the hot and the cold domains. Distributions of the activator for different times. The parameters used are: $\epsilon=0.05, \alpha=0.015, A=0.1$. The system is $20 \times 20$. The boundary conditions are periodic. 


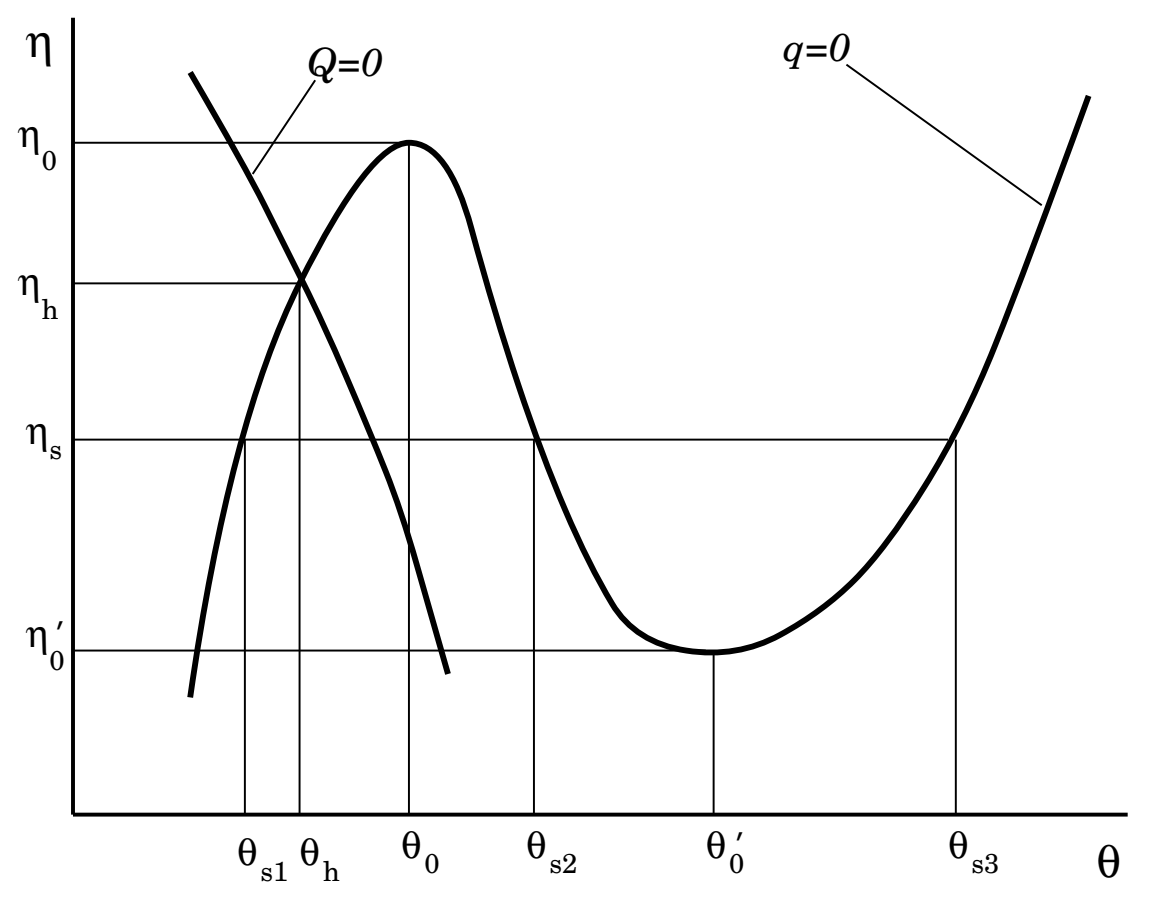




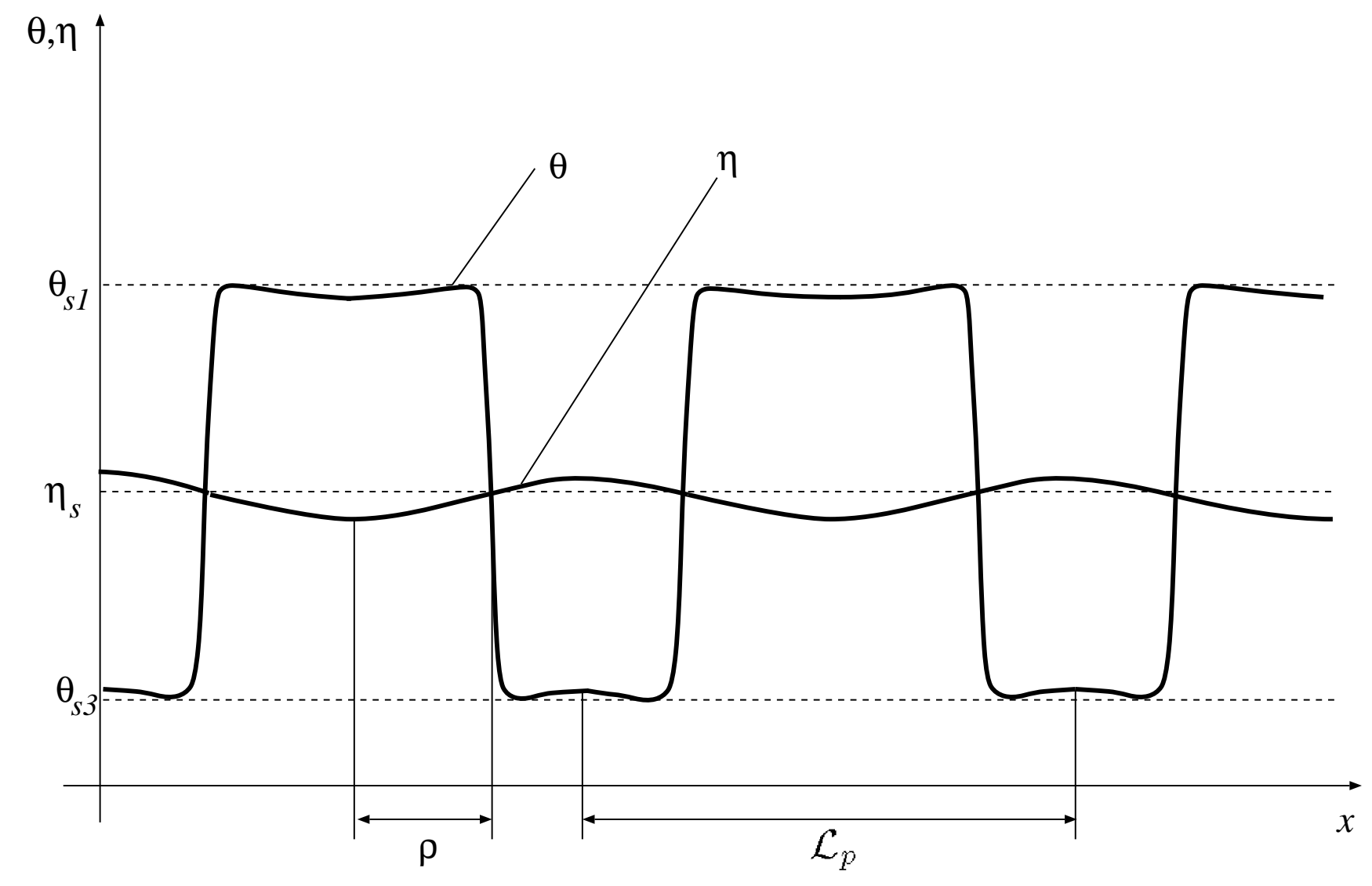




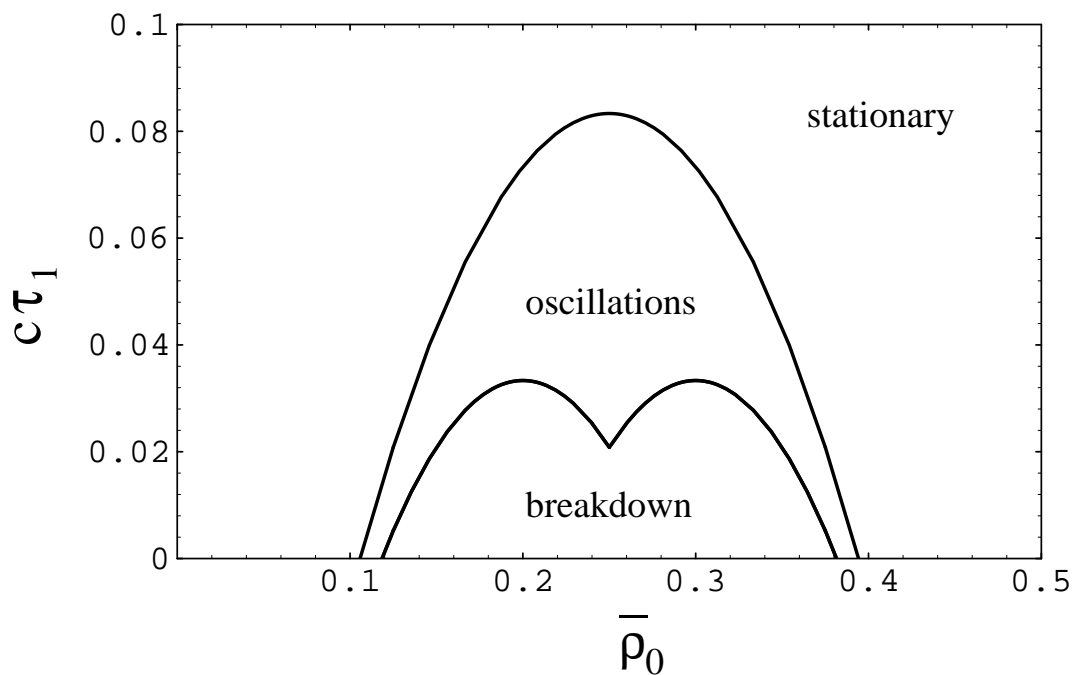




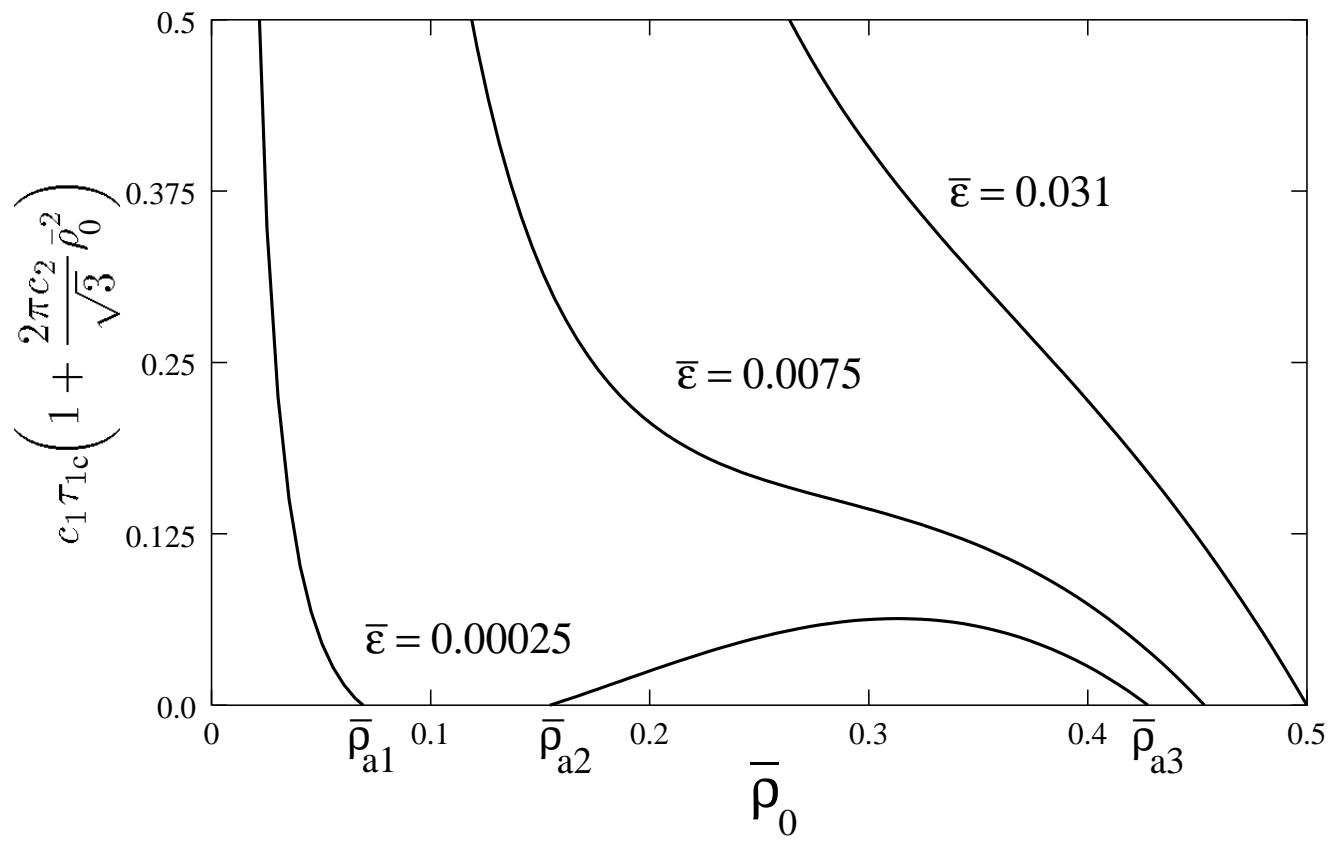



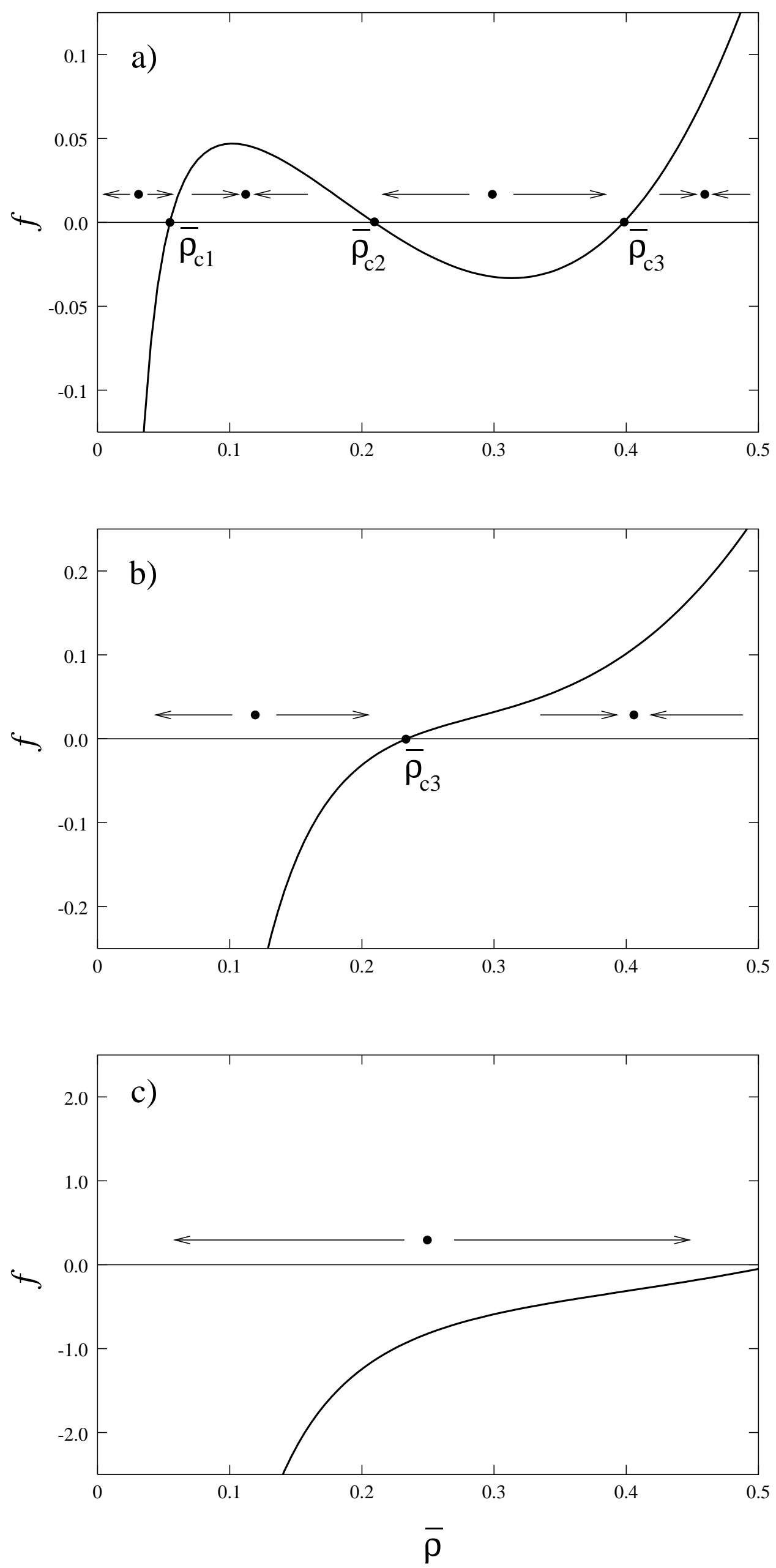


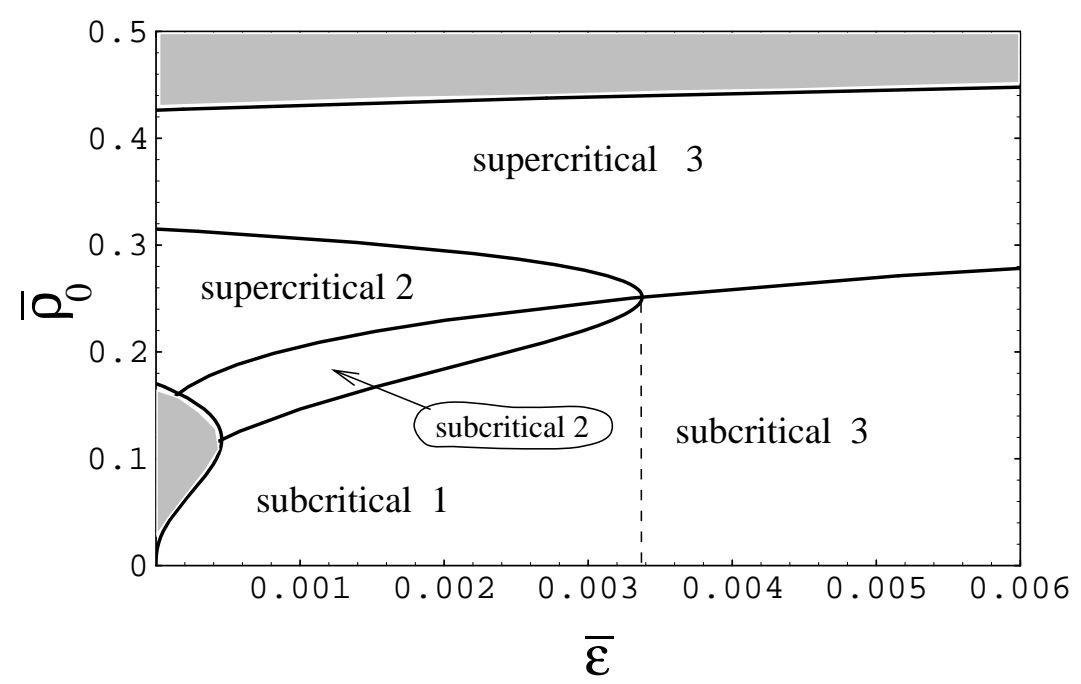




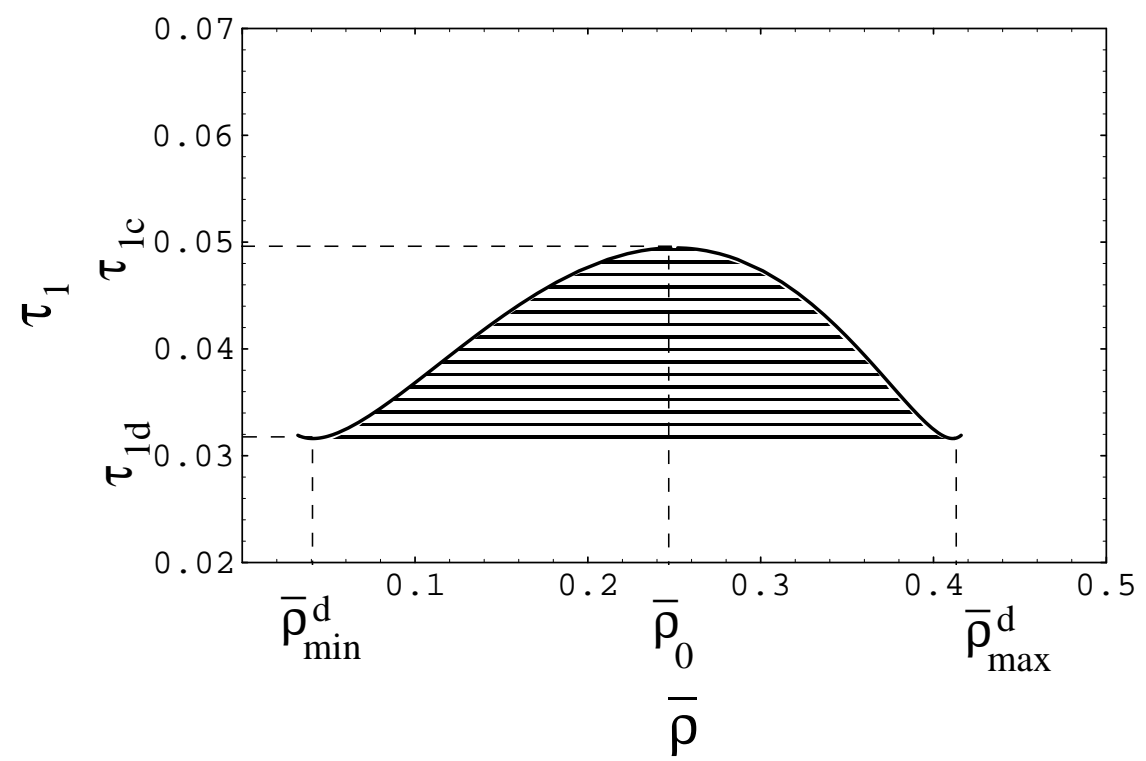



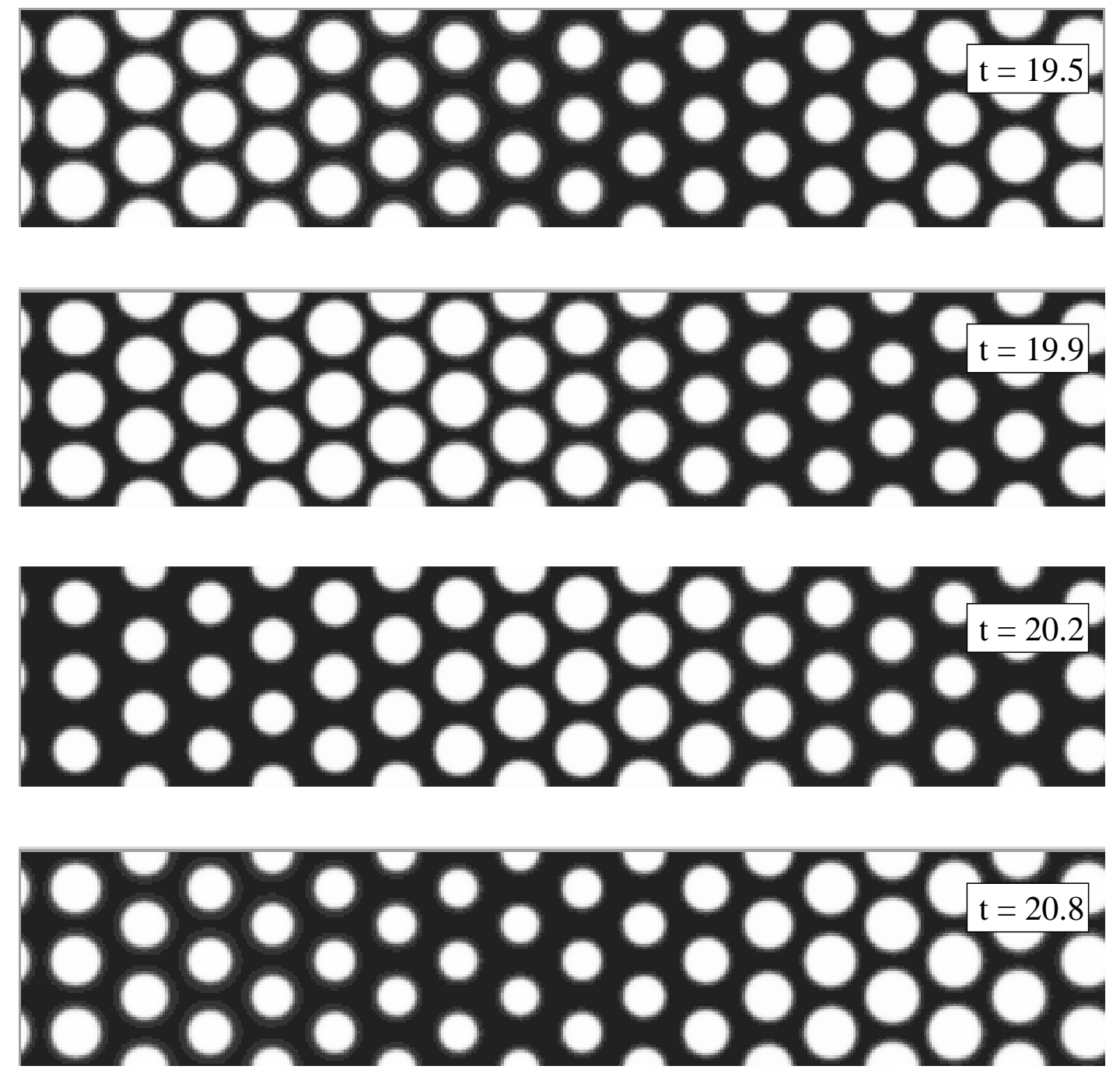
This figure "fig9.gif" is available in "gif" format from: http://arxiv.org/ps/patt-sol/9608005v1 
This figure "fig10.gif" is available in "gif" format from: http://arxiv.org/ps/patt-sol/9608005v1 
This figure "fig11.gif" is available in "gif" format from: http://arxiv.org/ps/patt-sol/9608005v1 
This figure "fig12.gif" is available in "gif" format from: http://arxiv.org/ps/patt-sol/9608005v1 